\title{
Concise and Efficient Synthesis of Calothrixin B
}

\author{
Drissa Sissouma, Lucie Maingot, Sylvain Collet* and André Guingant*
}

Université de Nantes, Nantes Atlantique Universités, CNRS, Faculté des Sciences et des Techniques, Laboratoire de Synthèse Organique (LSO), UMR CNRS 6513, 2, rue de la Houssinière - BP 92208 44322 NANTES Cedex 3

andre.guingant@univ-nantes.fr; sylvain.collet@univ-nantes.fr

\section{SUPPORTING INFORMATION}

Table of contents

Page

General experimental.................................................. S2

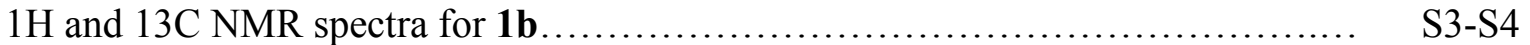

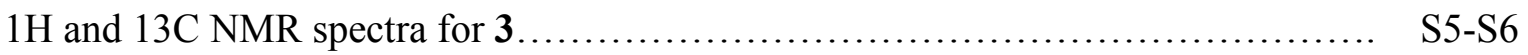

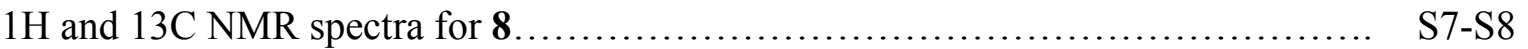

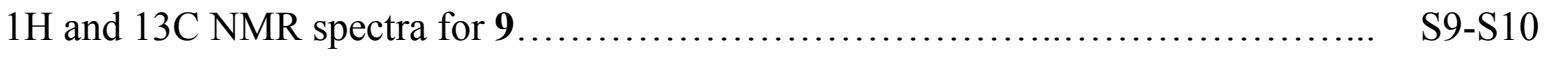

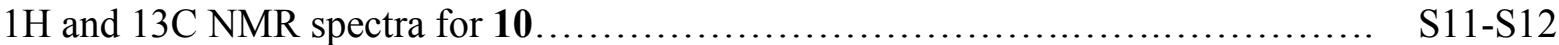

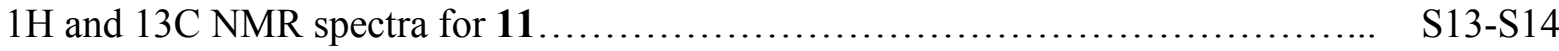

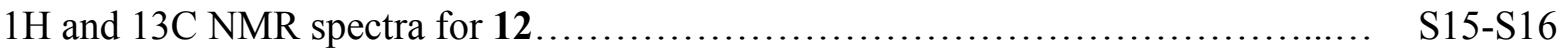

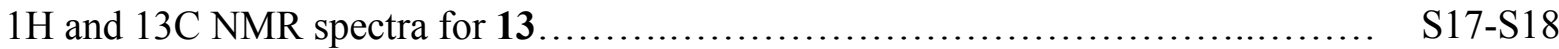

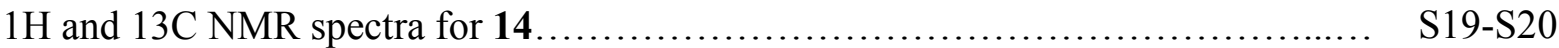

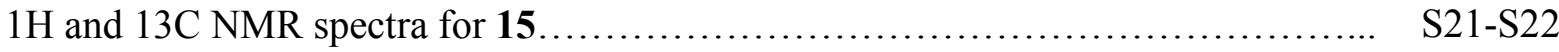

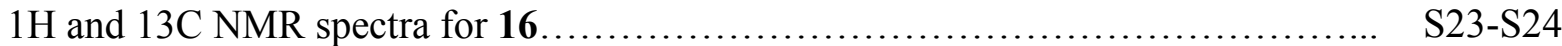

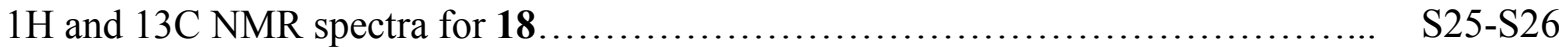


1H and 13C NMR spectra for 19

S27-S28

$1 \mathrm{H}$ and 13C NMR spectra for $\mathbf{2 1}$

$\mathrm{S} 29-\mathrm{S} 30$

1H and 13C NMR spectra for 22 .

S31-S32

1H and 13C NMR spectra for $\mathbf{2 3}$

S32-S34

1H and 13C NMR spectra for 24 .

S35-S36

General experimental. Melting points were determined using a Kofler block and are uncorrected. IR spectra were recorded neat or as $\mathrm{KBr}$ pellets. ${ }^{1} \mathrm{H}$ NMR spectra were recorded on spectrometers at 300 MHz or at $400 \mathrm{MHz} .{ }^{13} \mathrm{C}$ NMR spectra were recorded on spectrometers at $75 \mathrm{MHz}$ or at $100 \mathrm{MHz}$. All NMR spectra were run in deuteriated solvents with tetramethylsilane as the internal standard. $J$ Values are given in $\mathrm{Hz}$. The mass spectra were obtained in GC/MS mode (EI, $70 \mathrm{eV}$ ). Thin layer chromatography (TLC) was carried out on Kieselgel 60 F254 fluorescent plates. Flash chromatography was performed with silica gel $(40-63 \mu)$. Reactions carried out under an inert atmosphere refer to the use of argon or nitrogen. Diethyl ether, tetrahydrofuran (THF), toluene and benzene were dried by being distilled from sodium and benzophenone. Dichloromethane and acetonitrile were dried by distillation from calcium hydride. All other reagents were purified by distillation, the pressure being reduced if the boiling point of the compound was greater than $110^{\circ} \mathrm{C}$ at atmospheric pressure. 


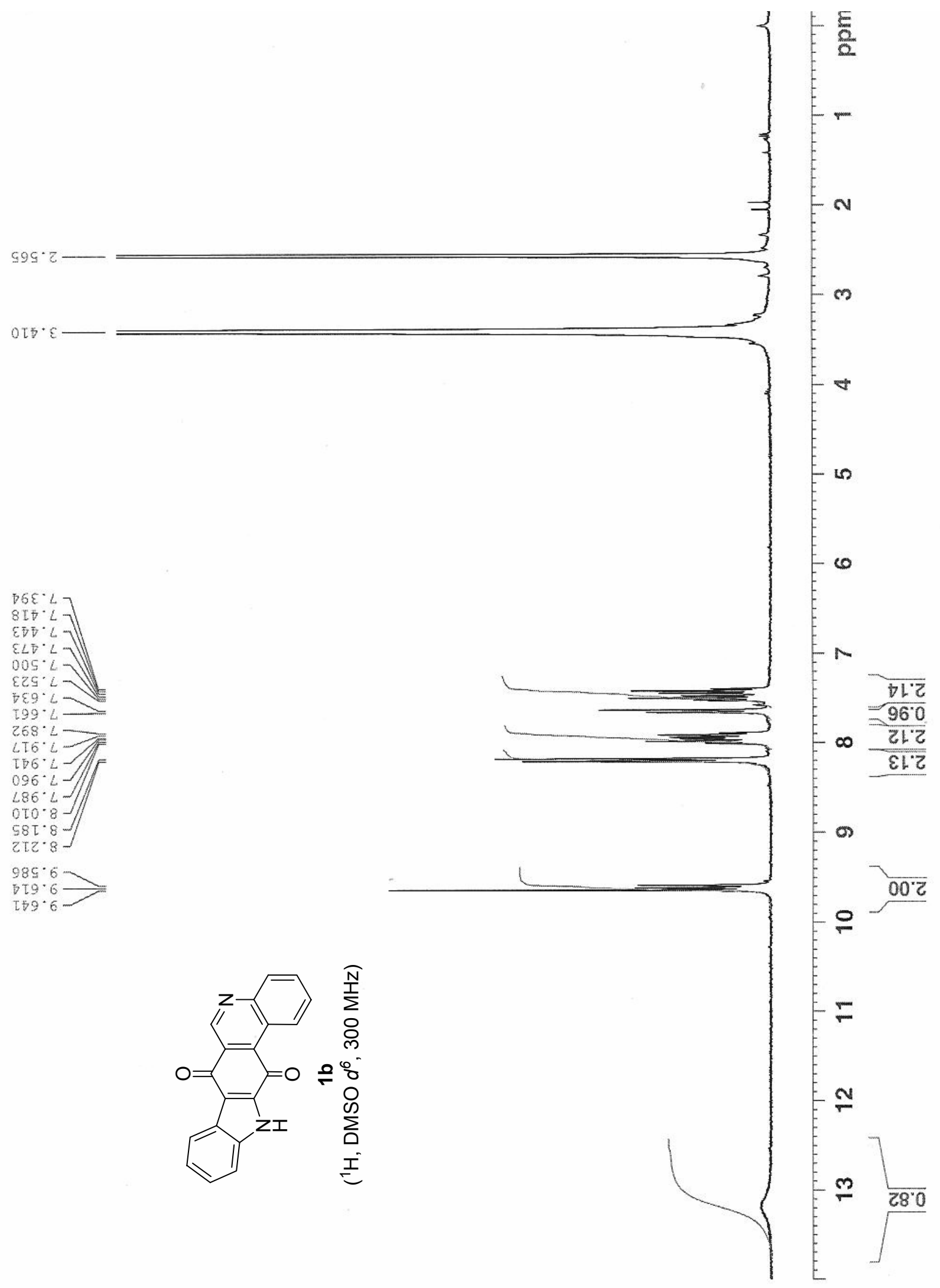




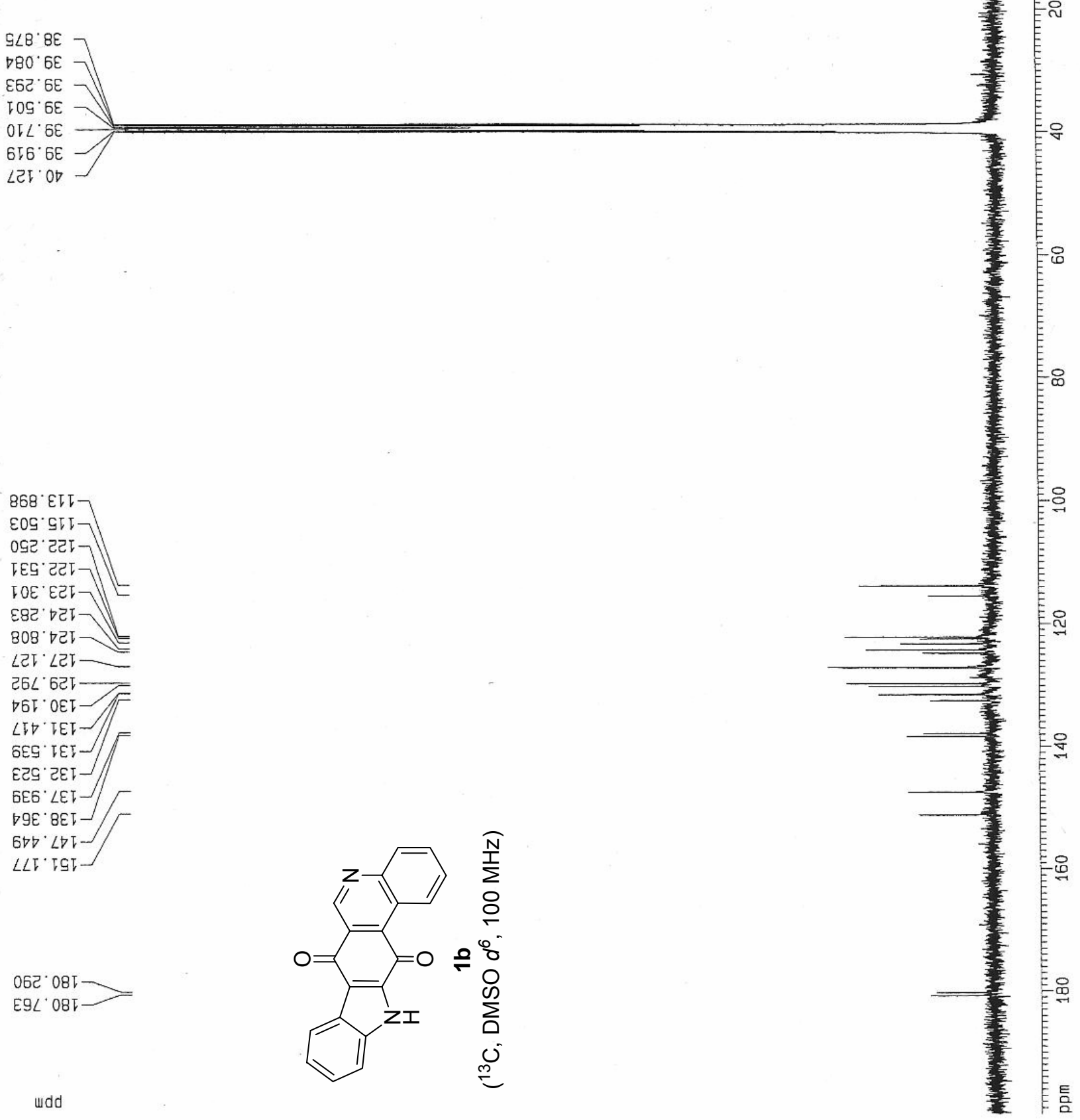




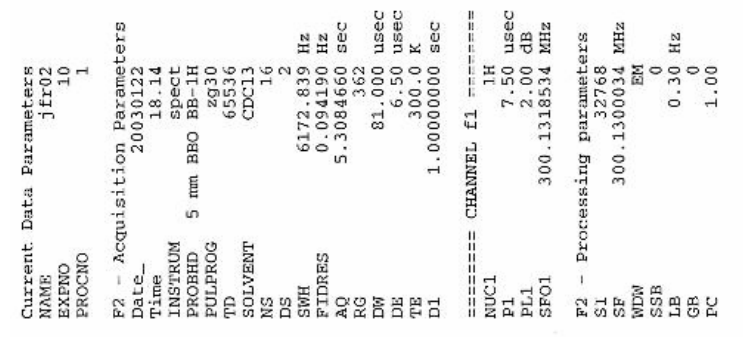

$000^{\circ} 0$
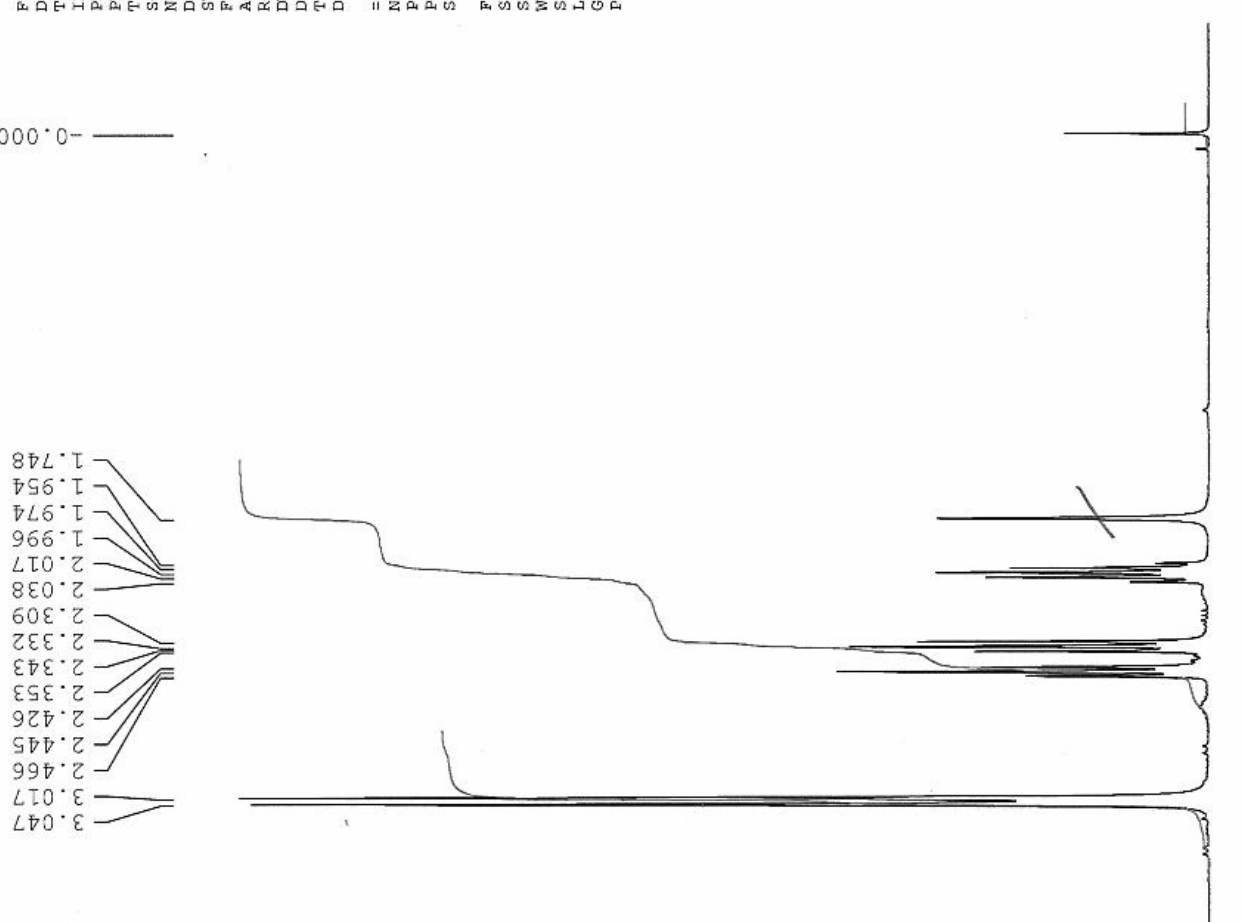

을

$\angle Z D \cdot S$
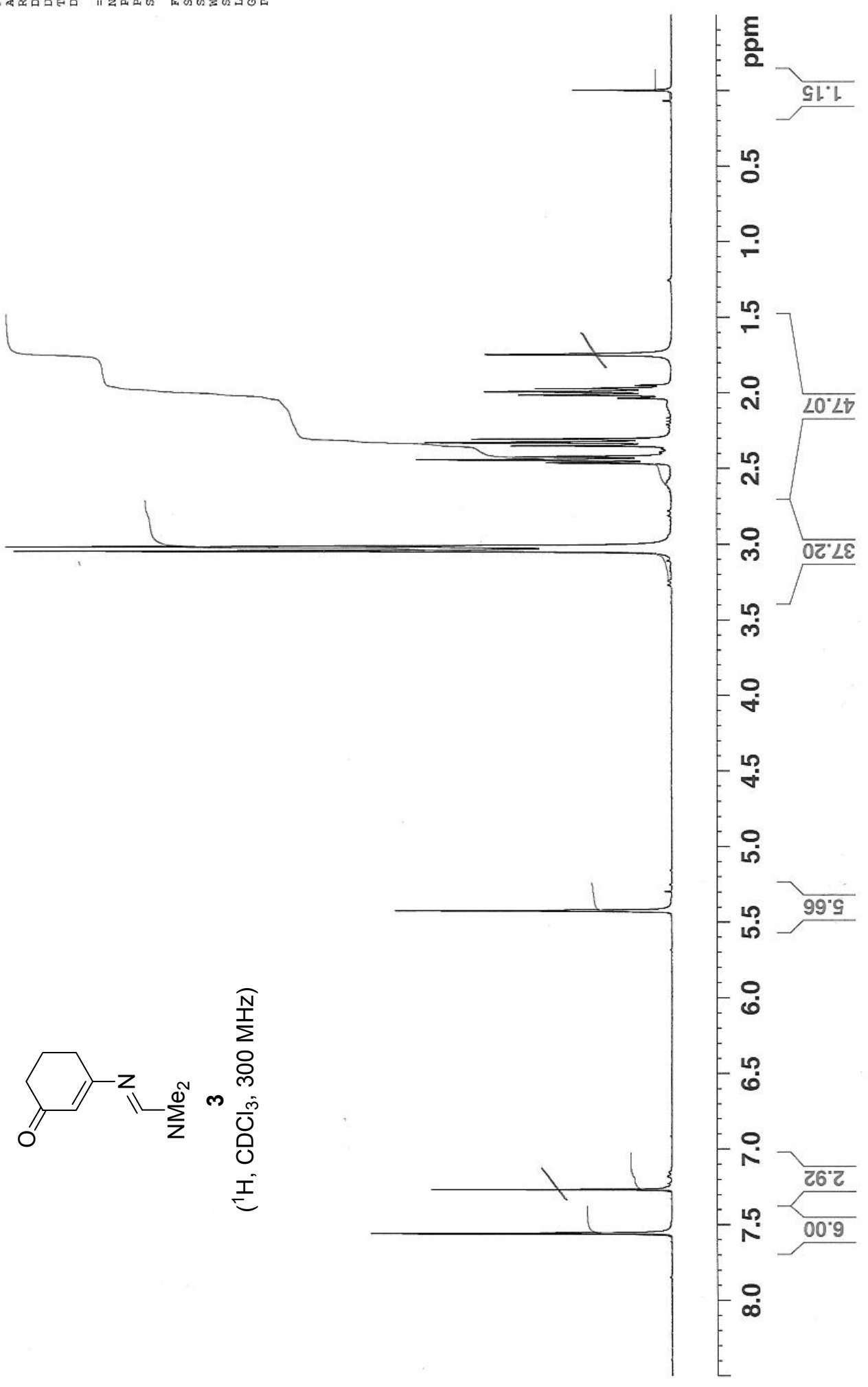
209025

ELCro 0

$8898^{\circ} 68$

$279298-$

948800

387992
009124

$00912 \mathrm{~L}-$
$8608 \mathrm{~L}-$

8229011

88\%0) 391

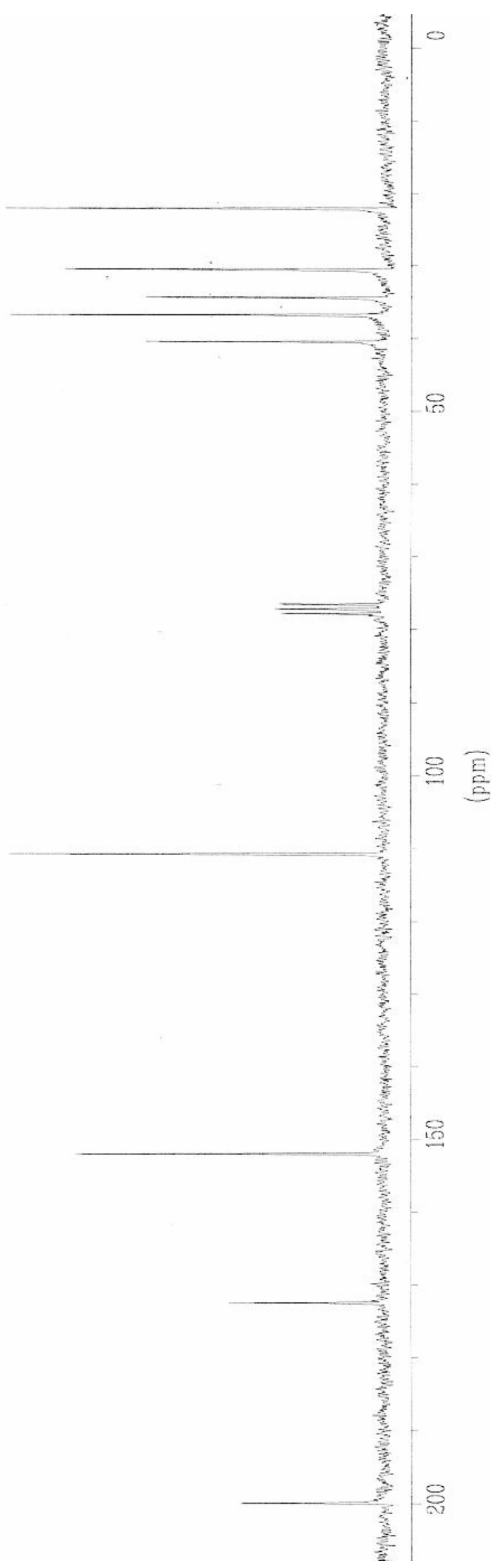

$1128661-$ 


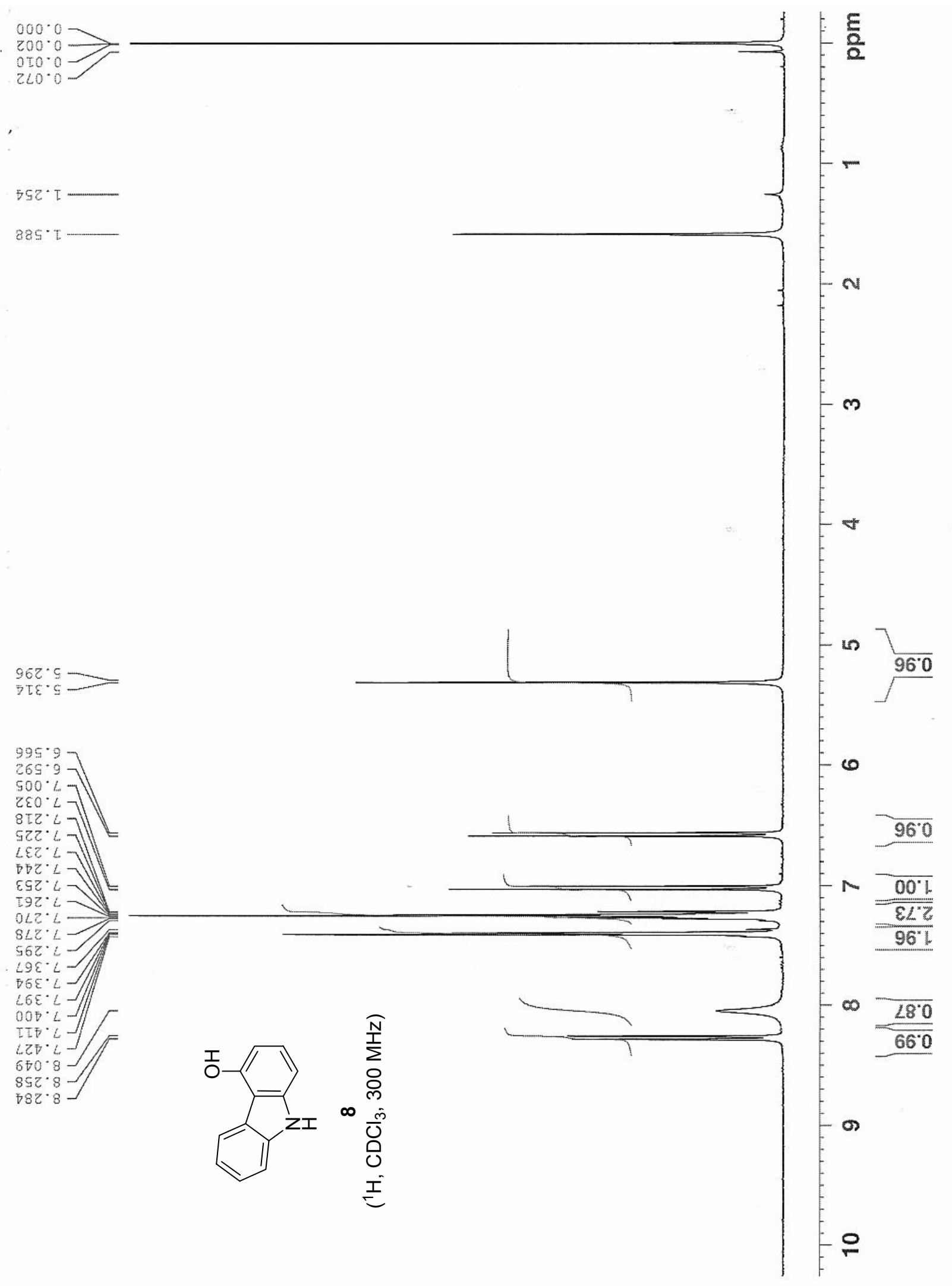



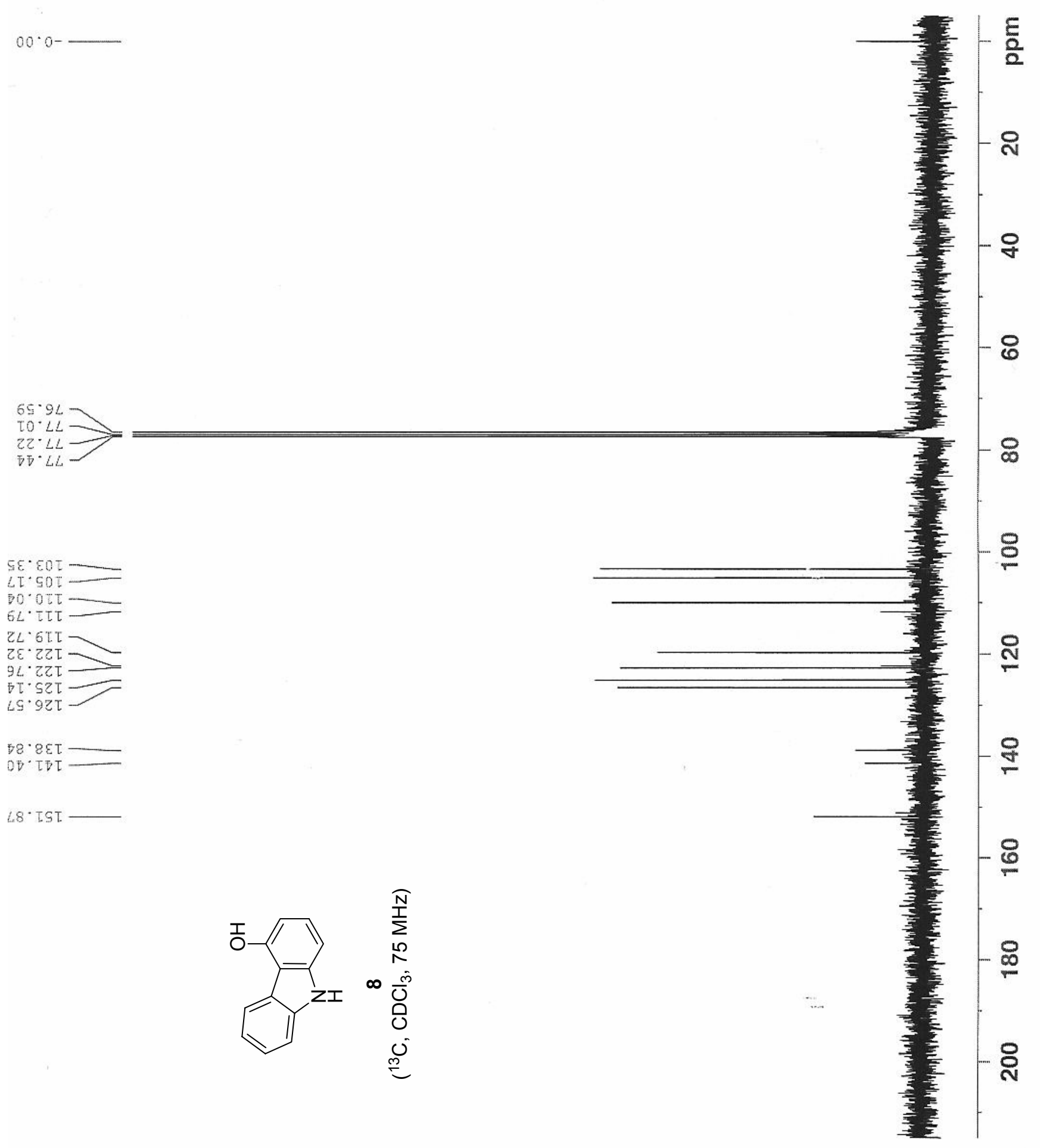


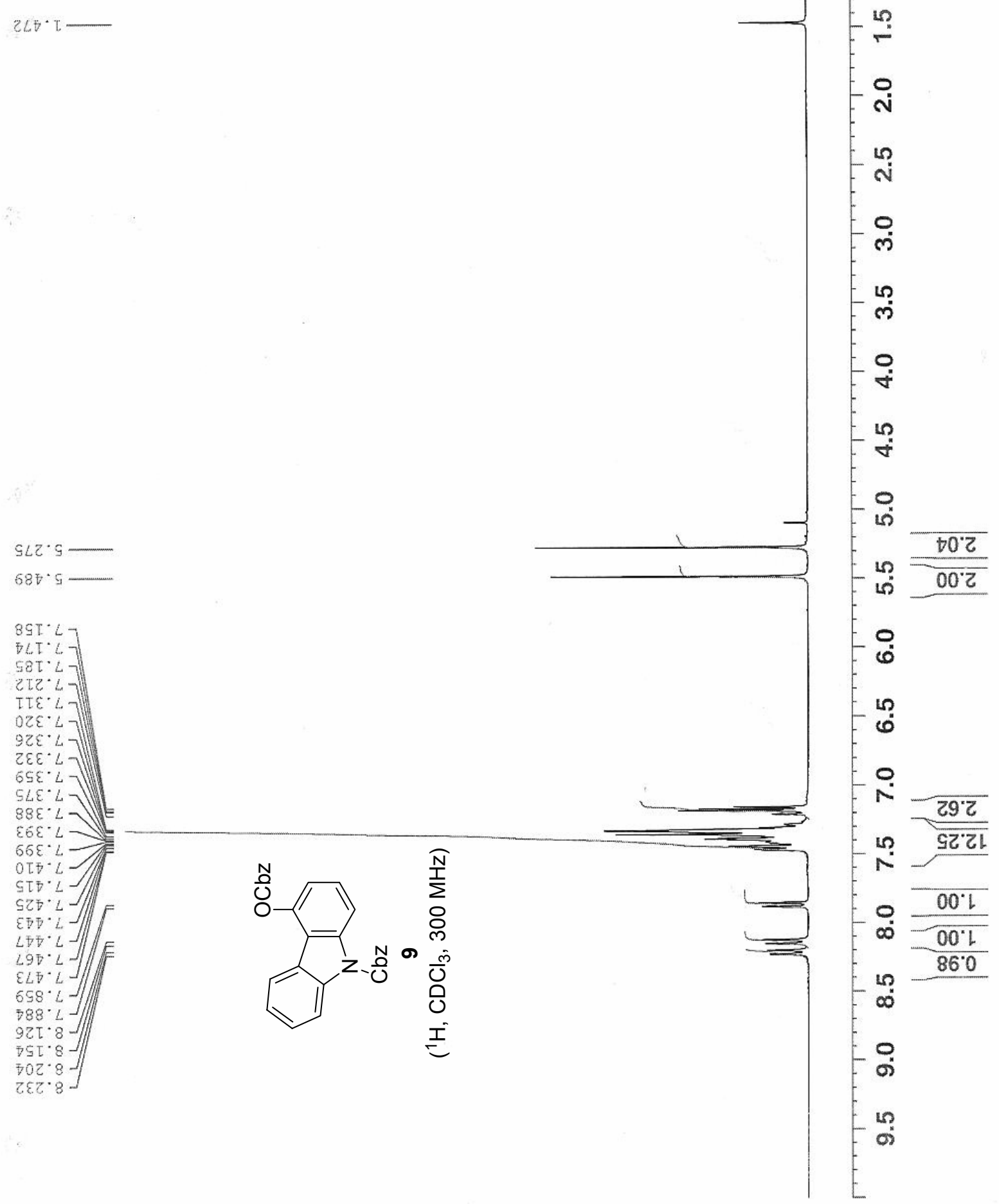




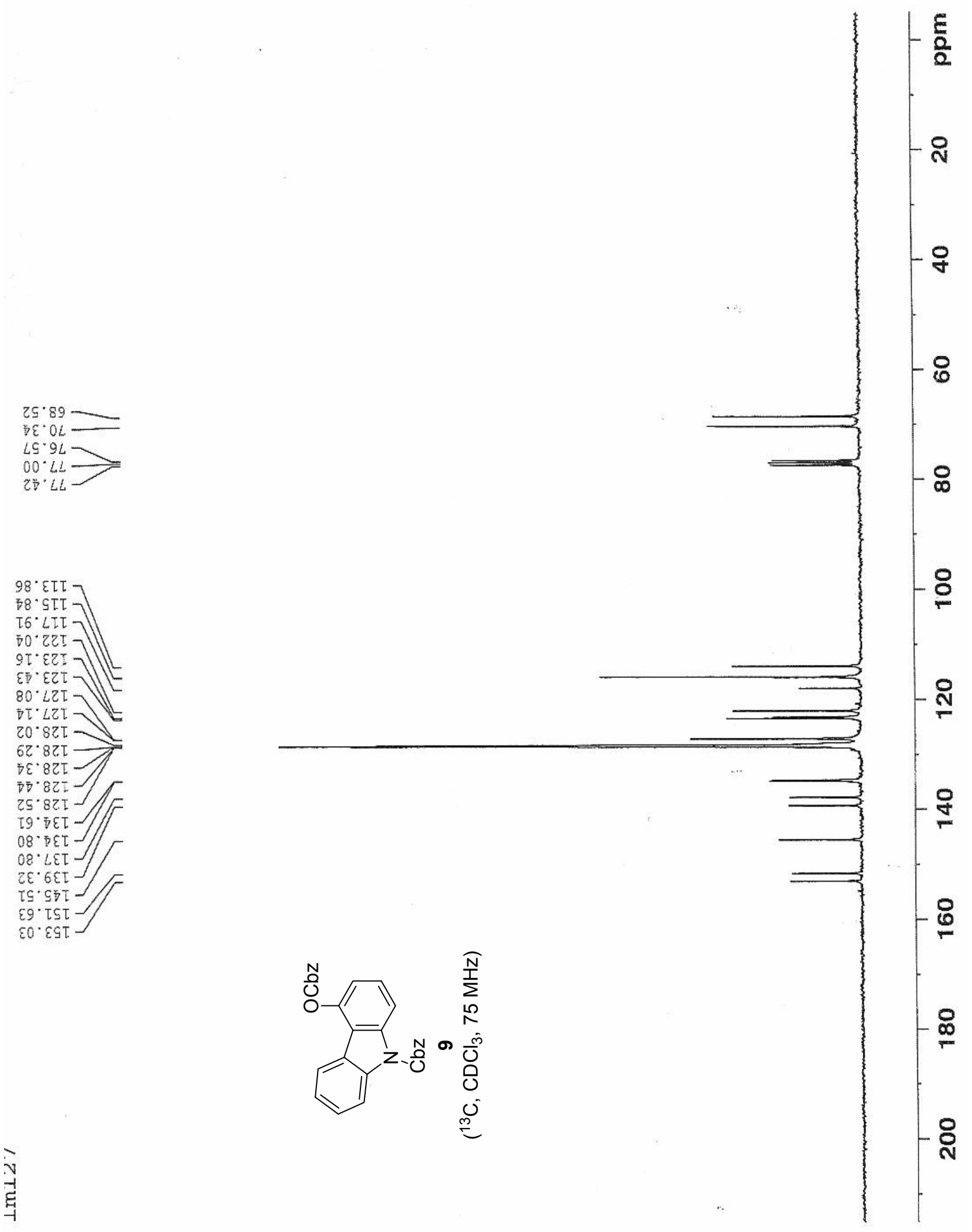




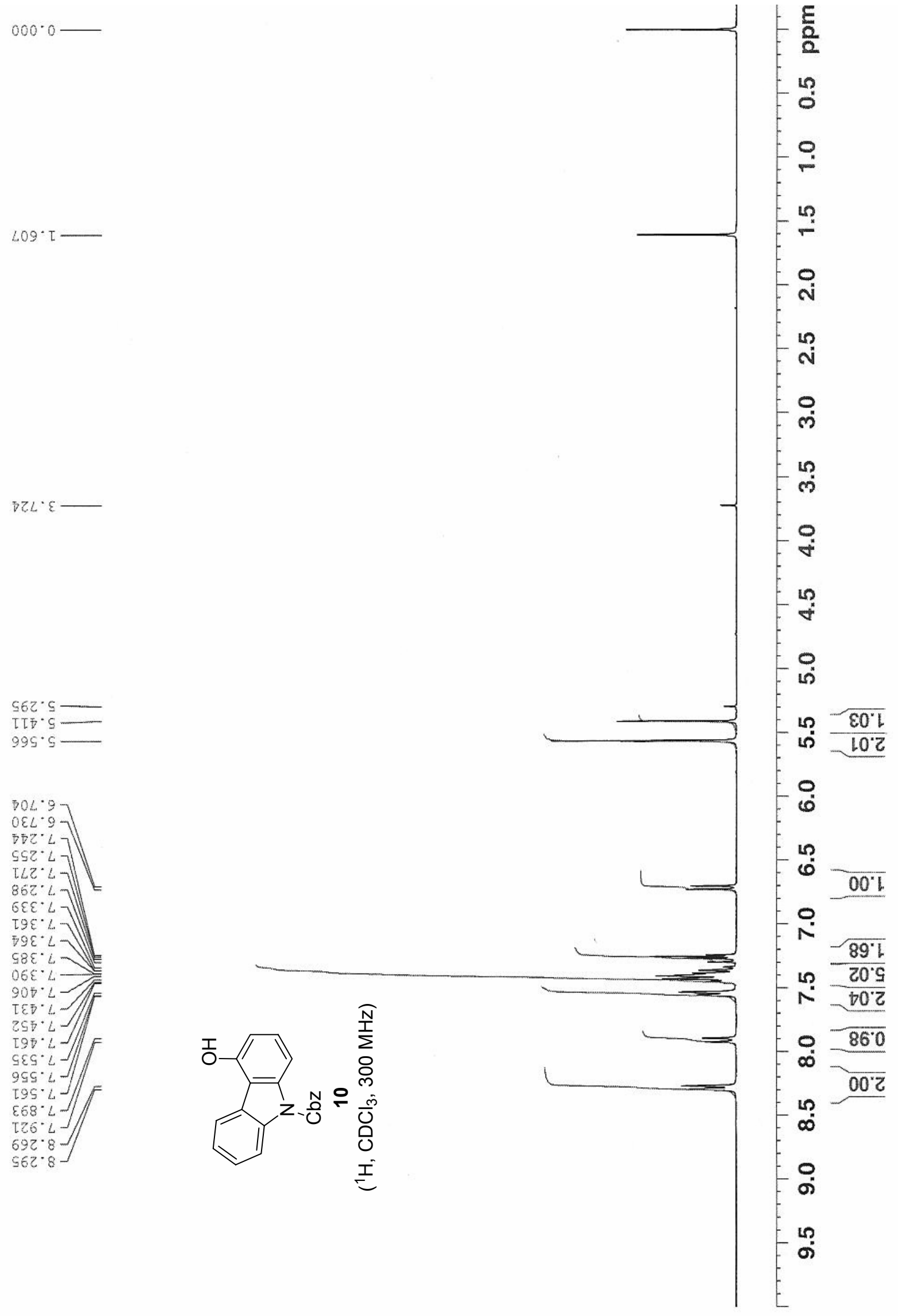




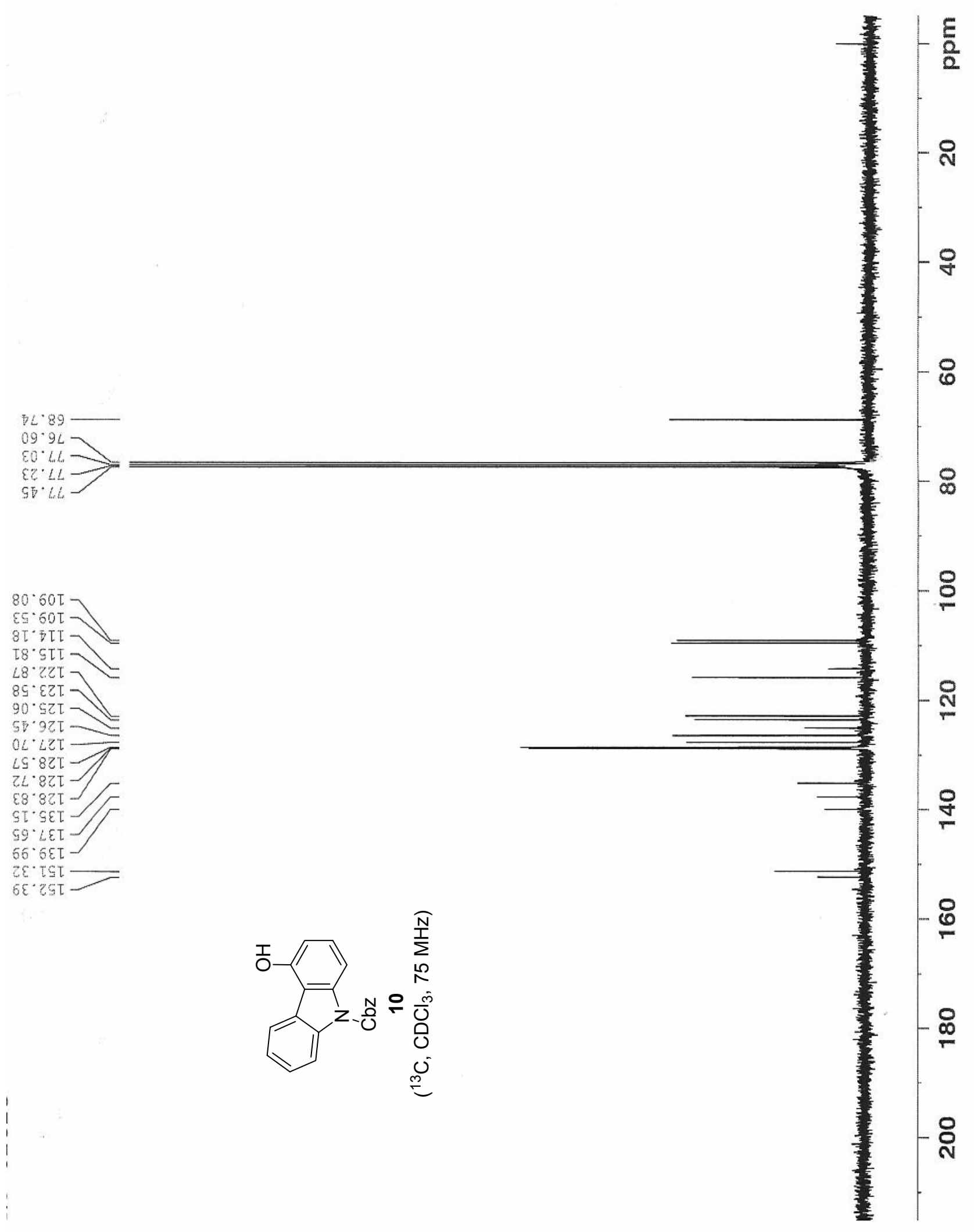




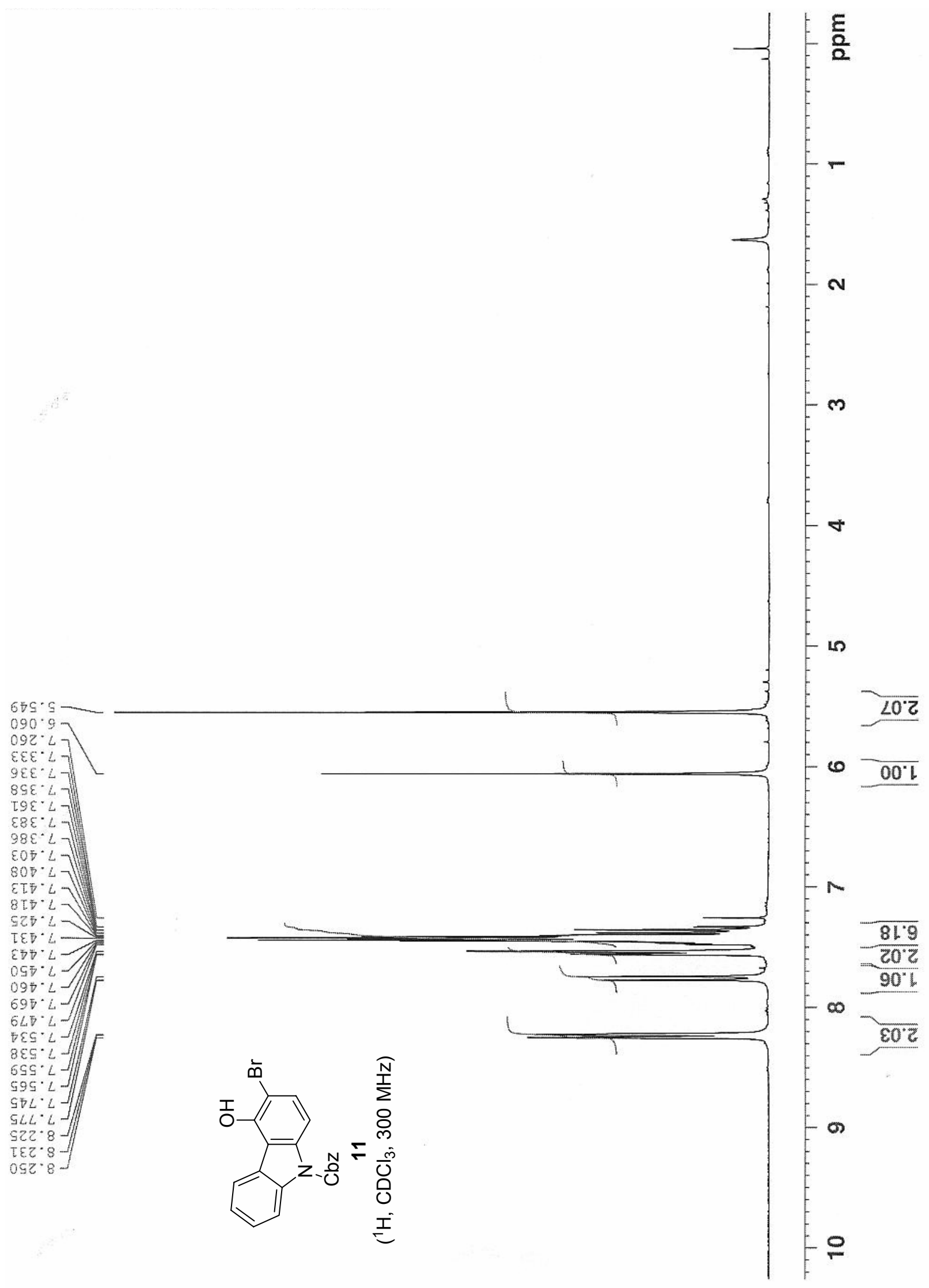




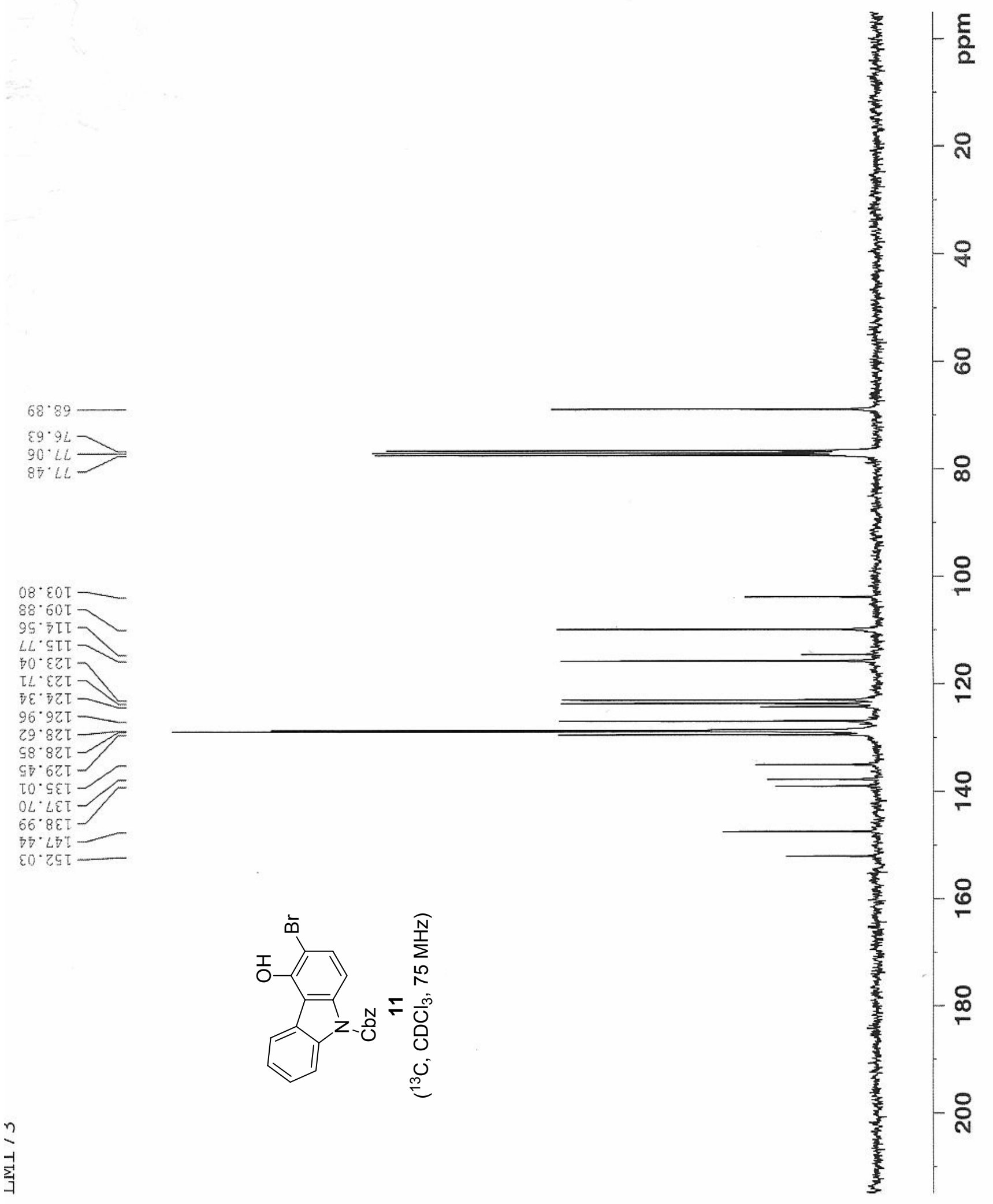


โ85. โ

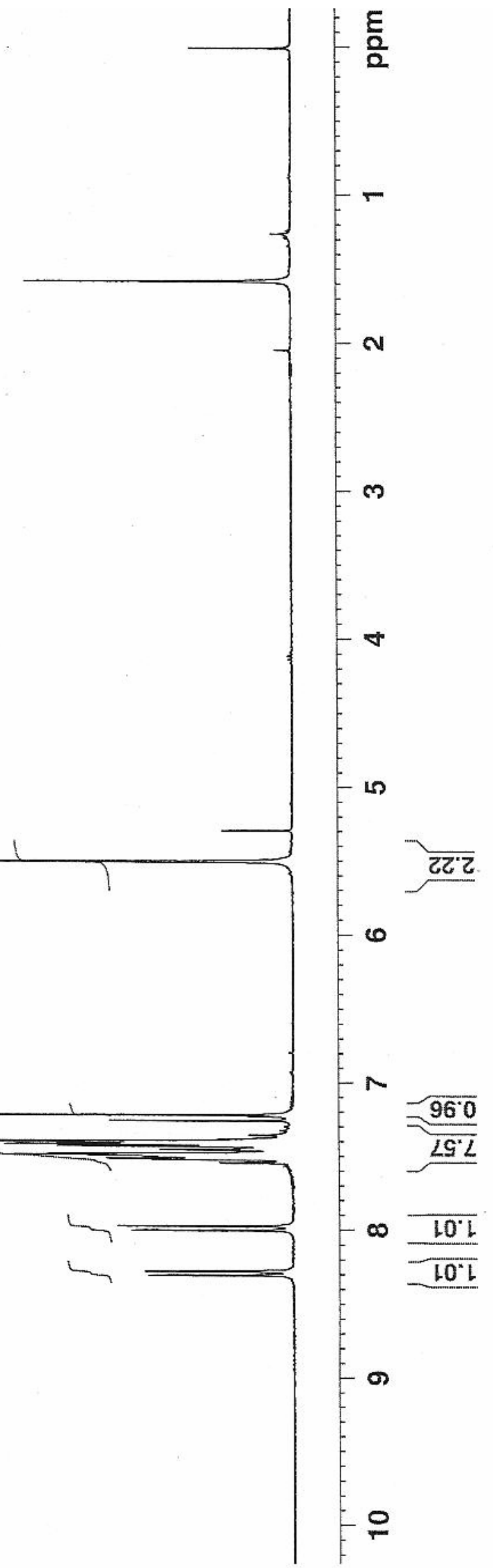




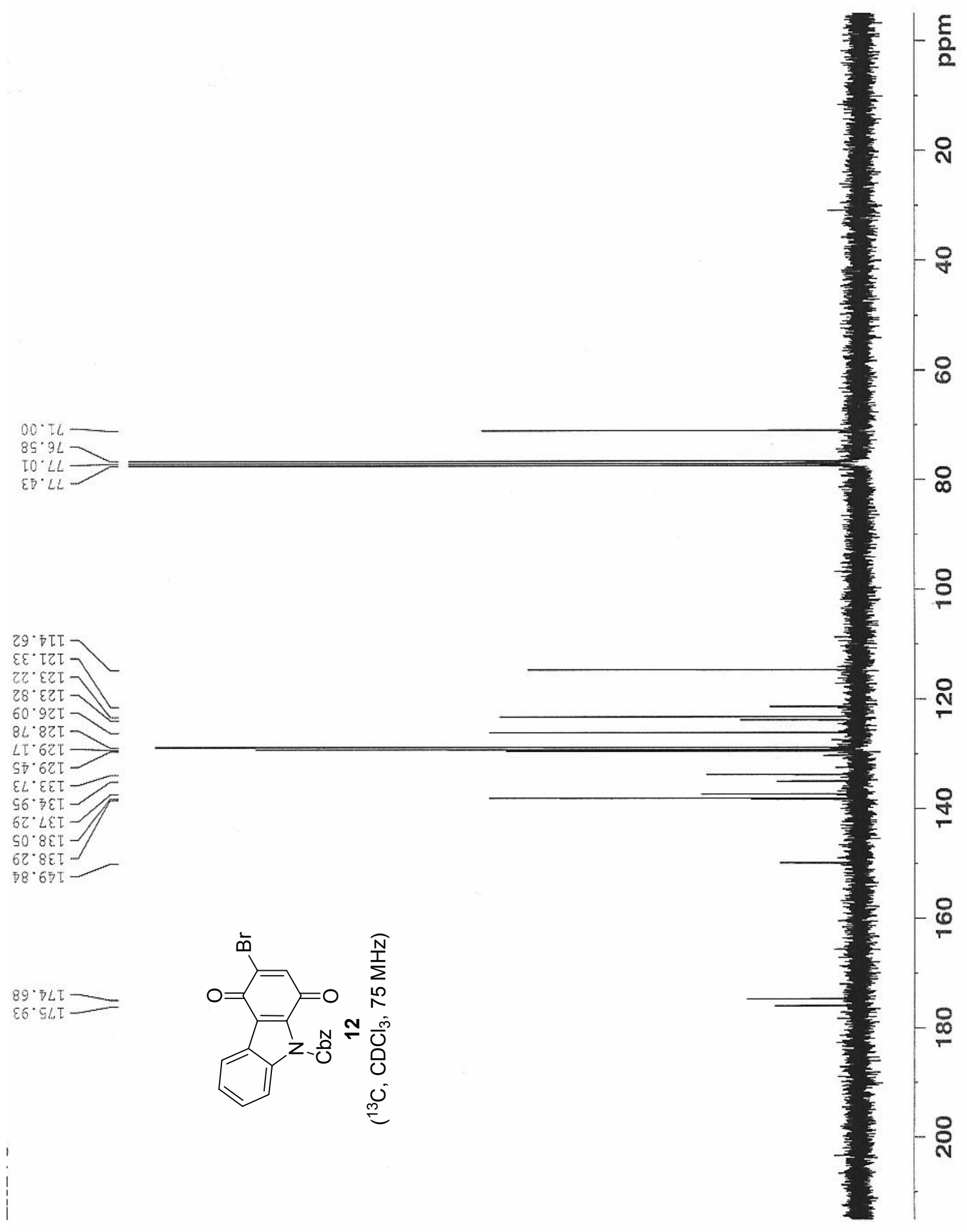



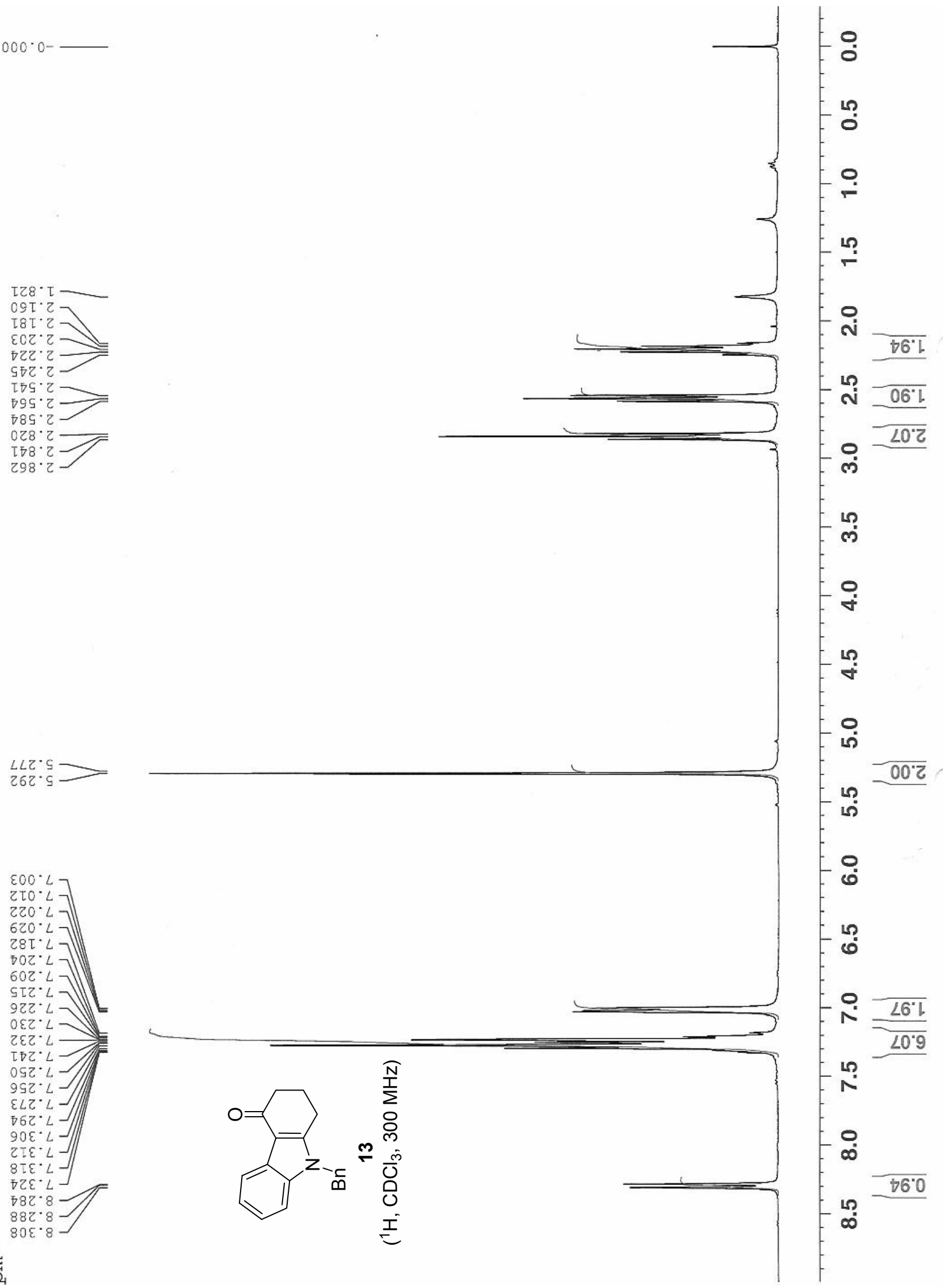


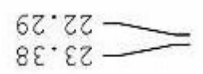

$68^{\circ}$ LE

$66 \cdot 97-$

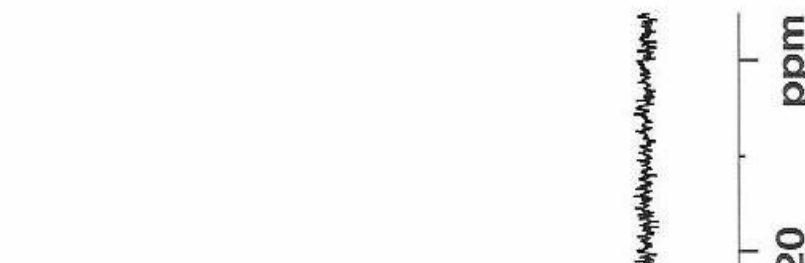

$\angle 9 \cdot 9 L-$

$60^{\circ} \mathrm{LL}$

T与 $L L-$

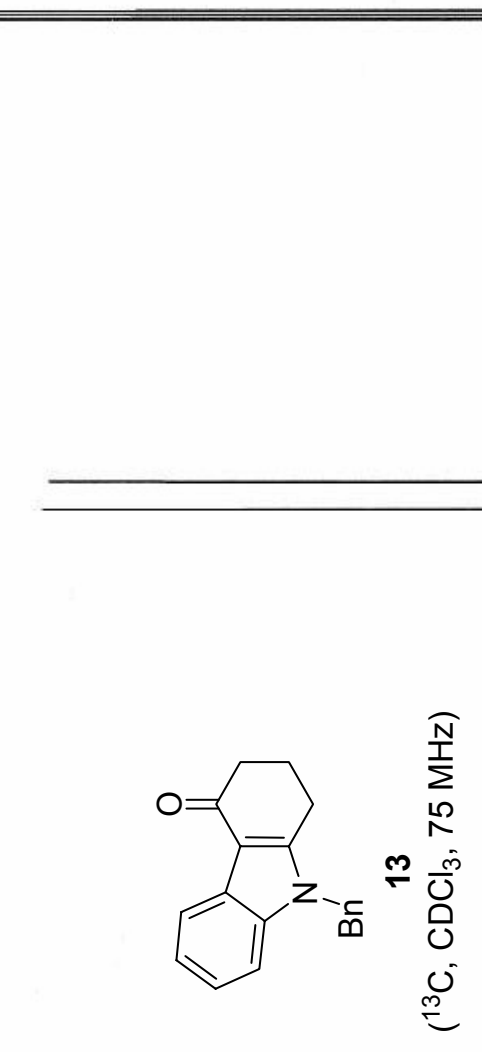

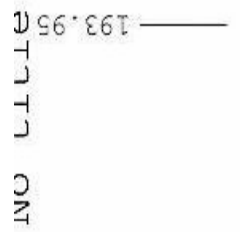

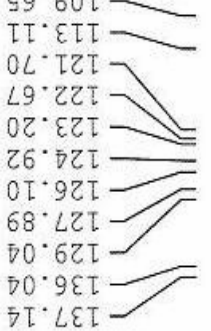

$\varepsilon 8^{\circ}$ โร

$\frac{\varepsilon}{2}$

ก

움

8

이

음

ำ

운

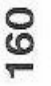

$\stackrel{\infty}{\sim}$

ลั 


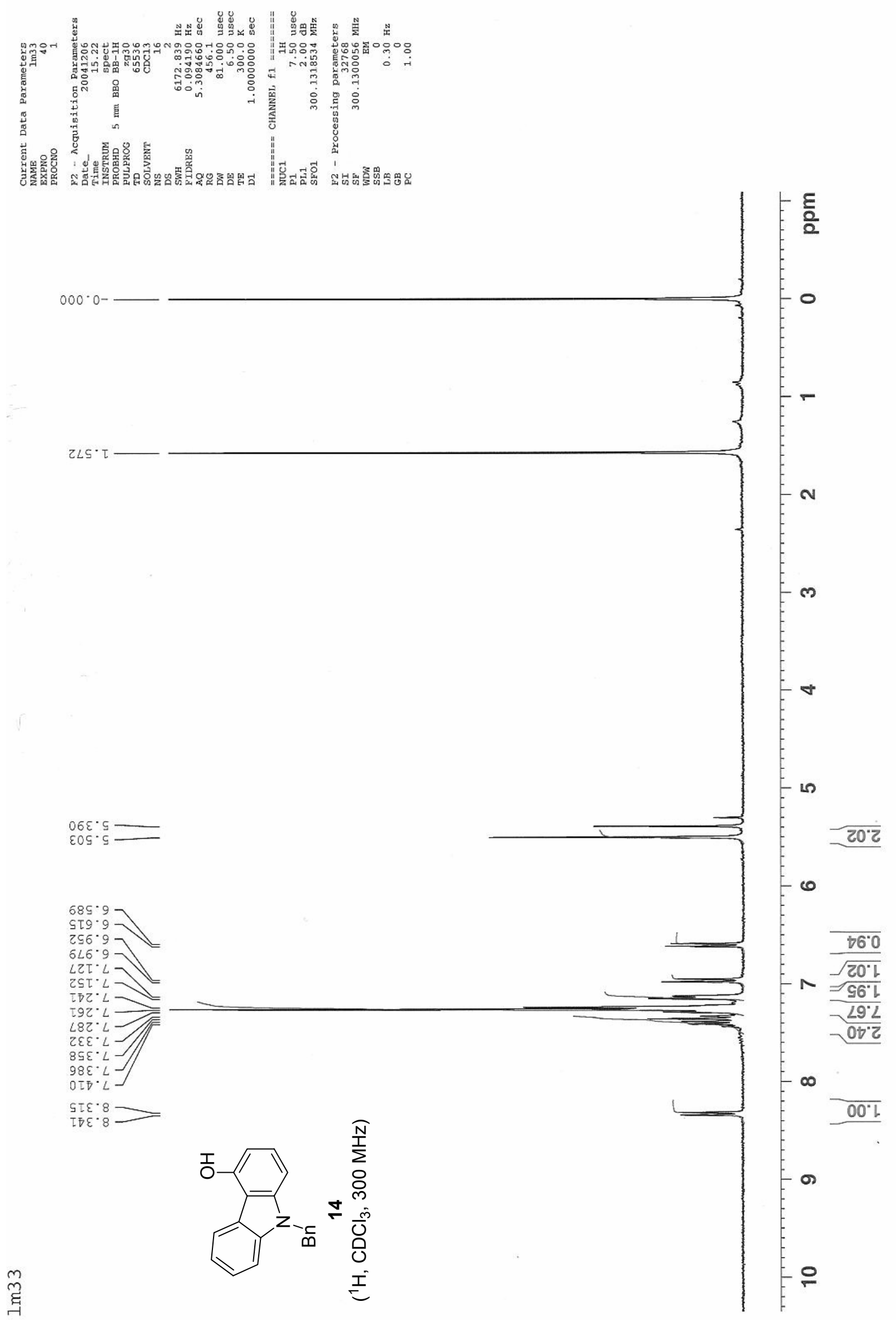


$9 L \cdot 97$

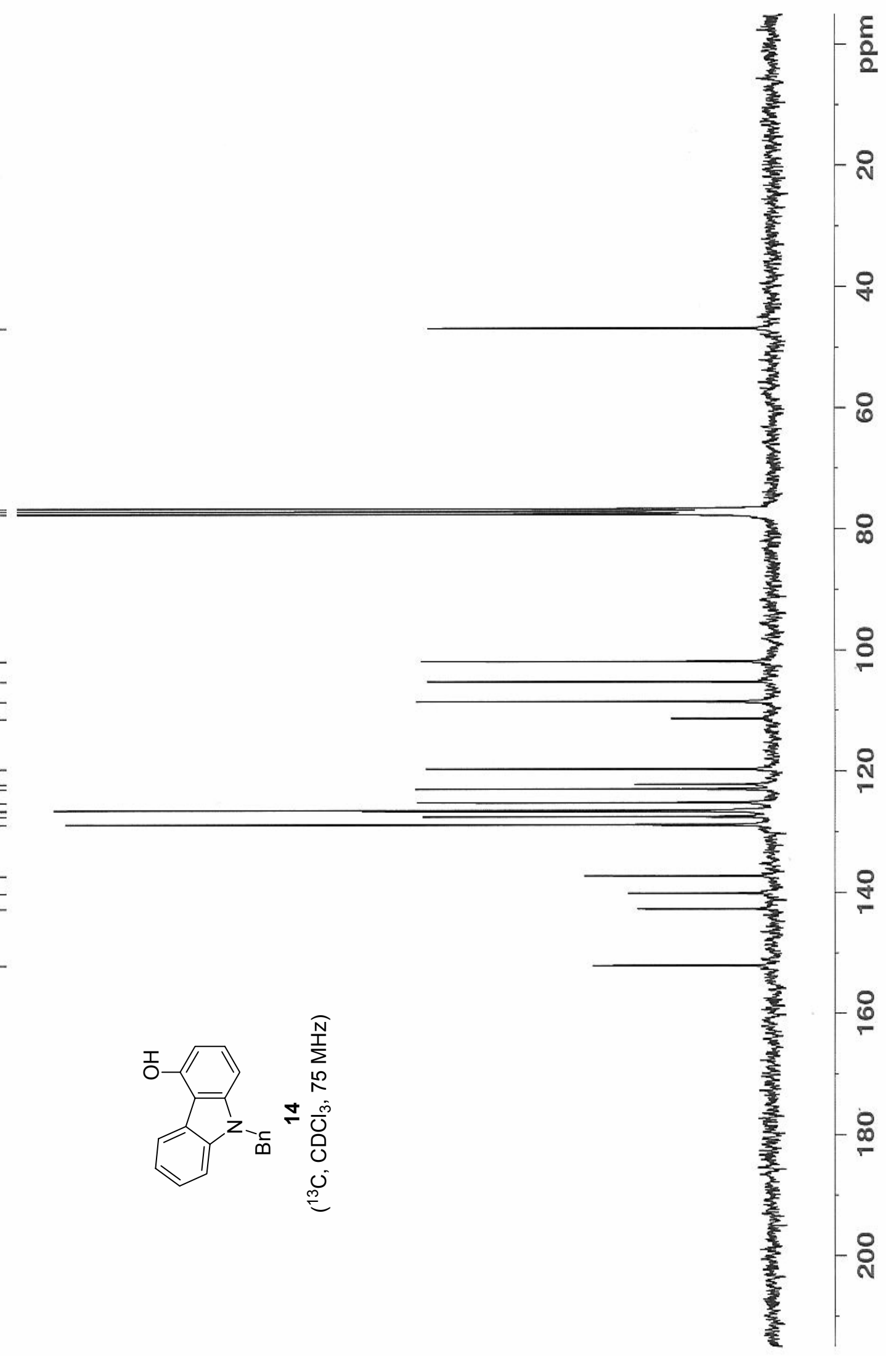

$99^{\circ} 9 \mathrm{LL} \longrightarrow$
$00^{\circ} \mathrm{LL} \longrightarrow$ 


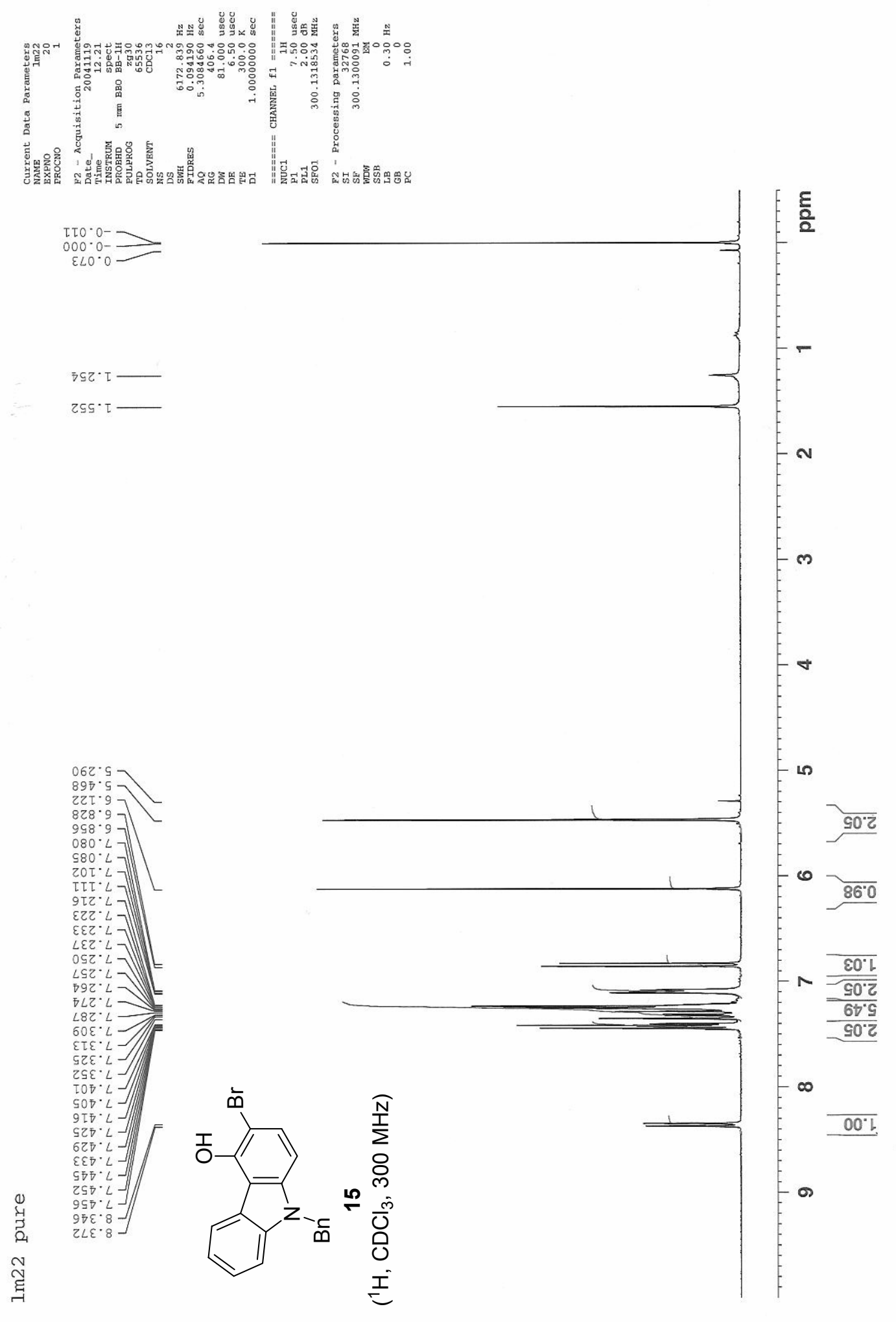




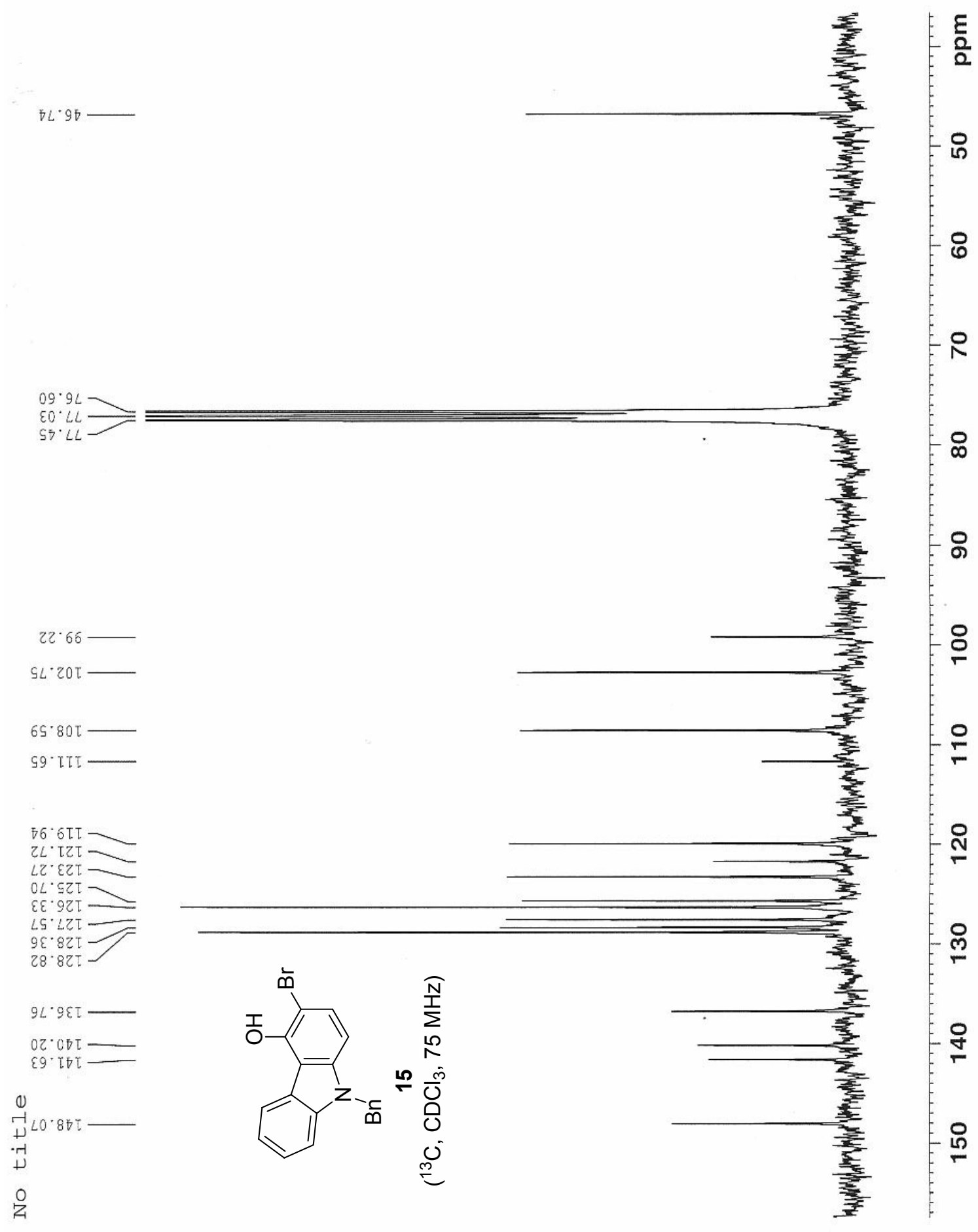




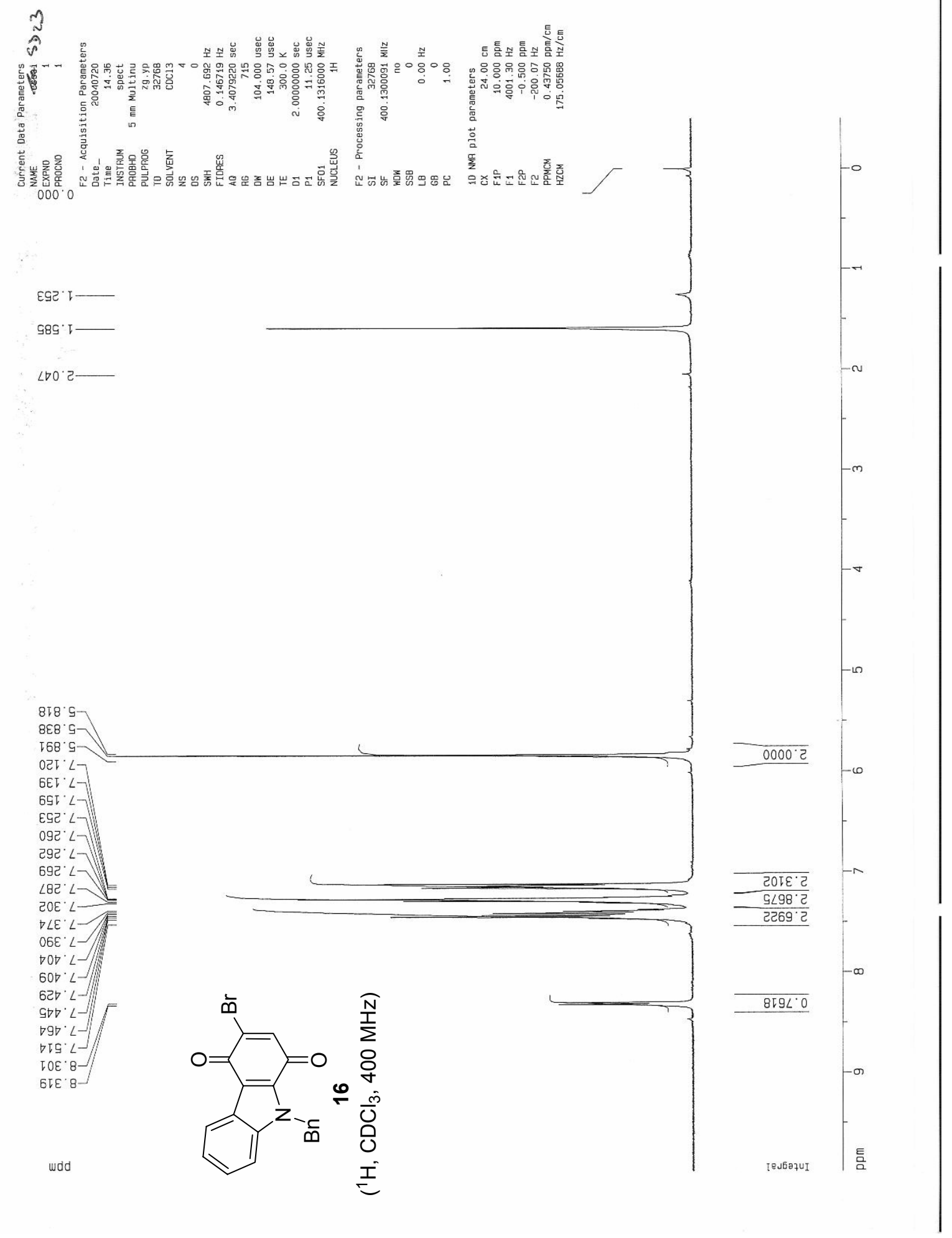


틍 틀소 틈소 흠소

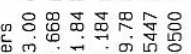

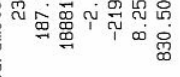

$\left.88 \varepsilon^{\circ} 8\right\rangle$

Et8. 9

I9I' $\angle L$
$8 \angle V^{\circ} \angle L$

OL 9 LI

घ9E. हटा-

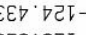

9टE जटโ

968.9टा-

$9 E L \angle Z T-$

$6 \varepsilon T^{\circ} 8 \mathrm{CL}-$

$9200^{\circ} 621-$

ОLट. घEा-

$620.9 E 1$

GOD' $\angle E T-$

IGC' GET-

gSE ODT-

$\tau \angle I \cdot G \angle I-$

$\angle 0{ }^{\circ} 8 \angle T=$

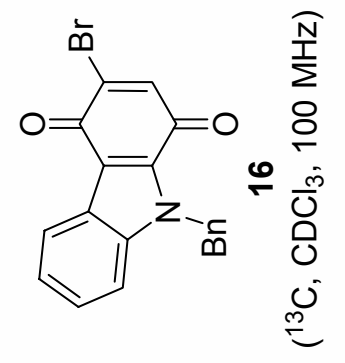

wdd 


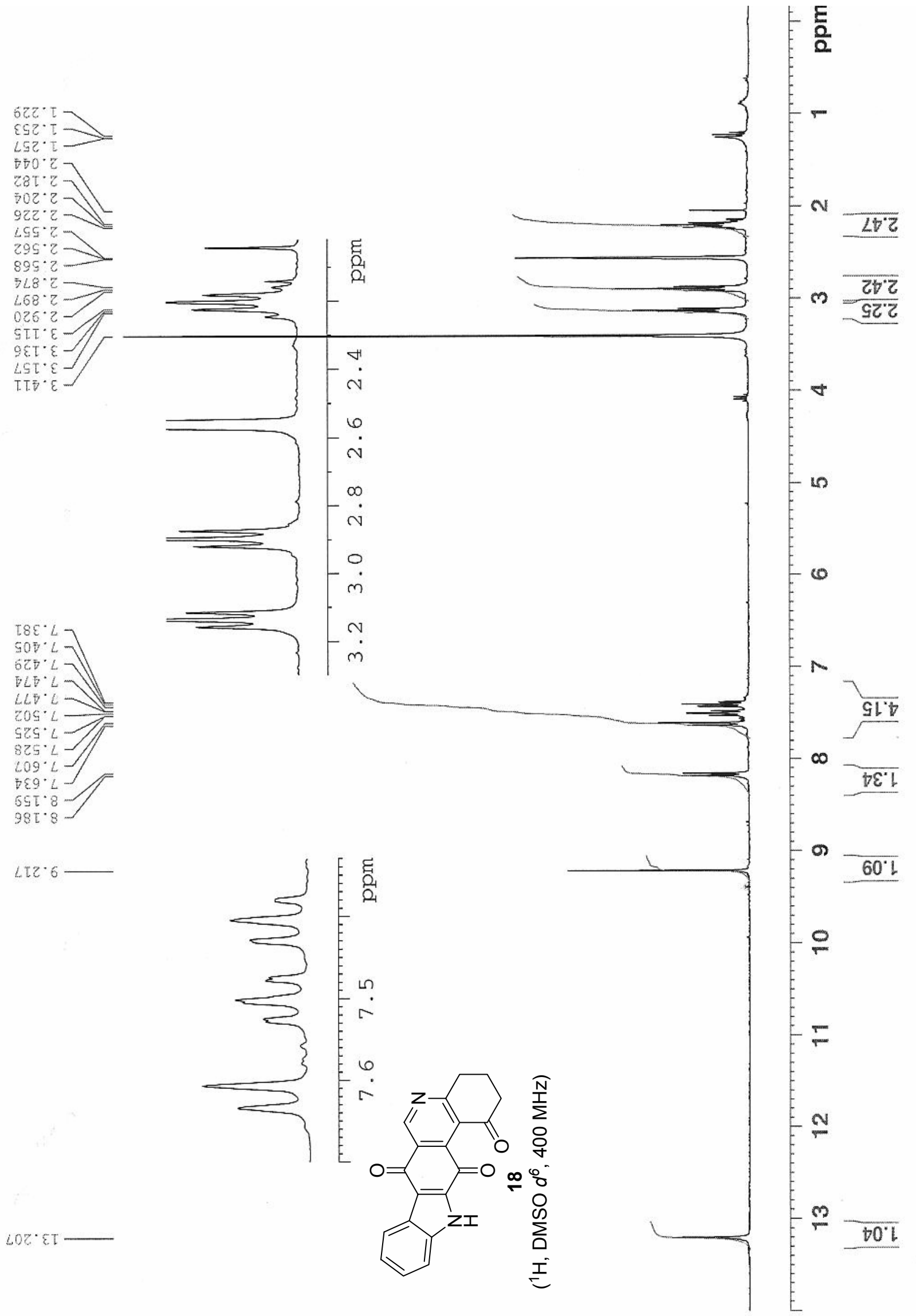




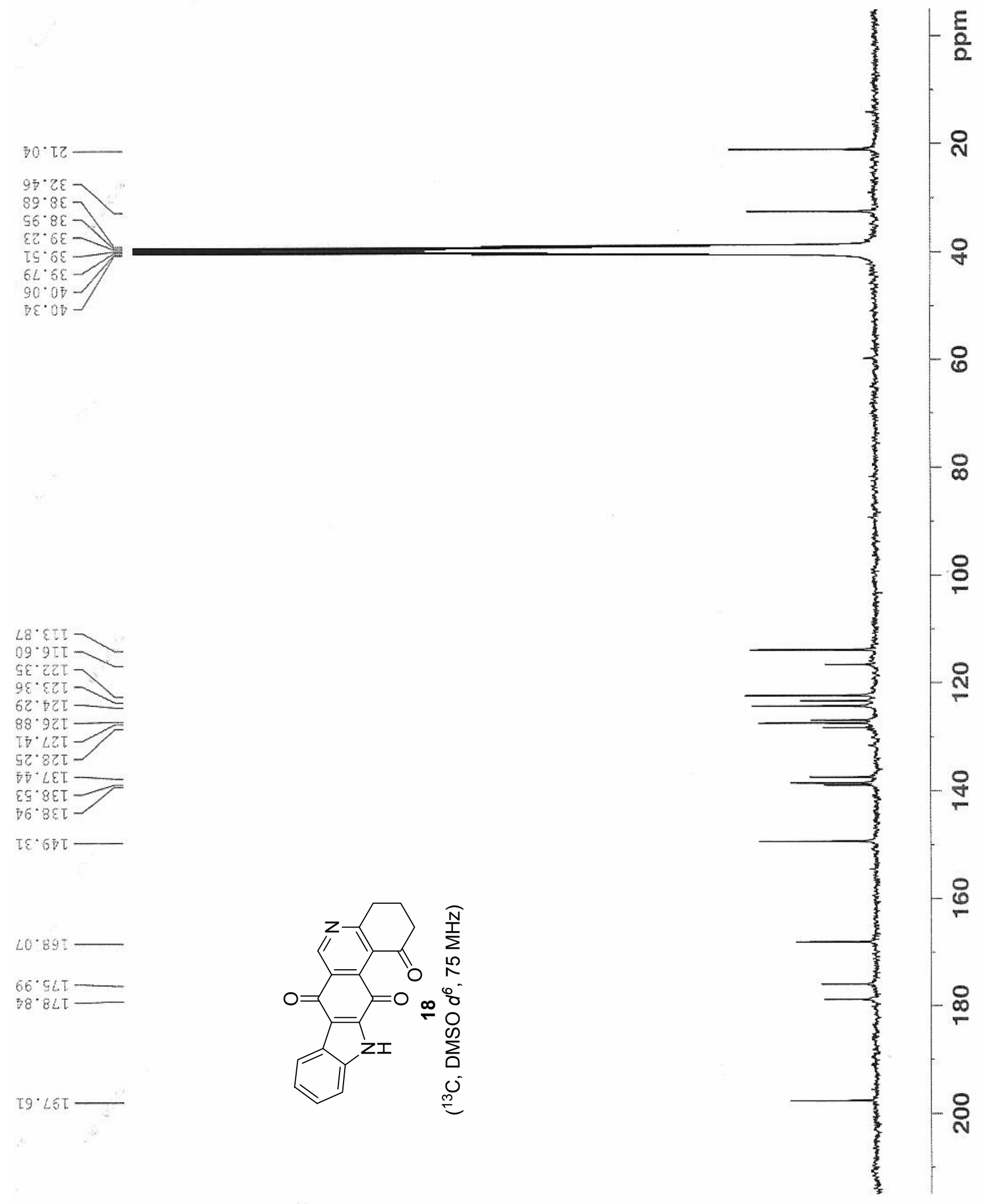




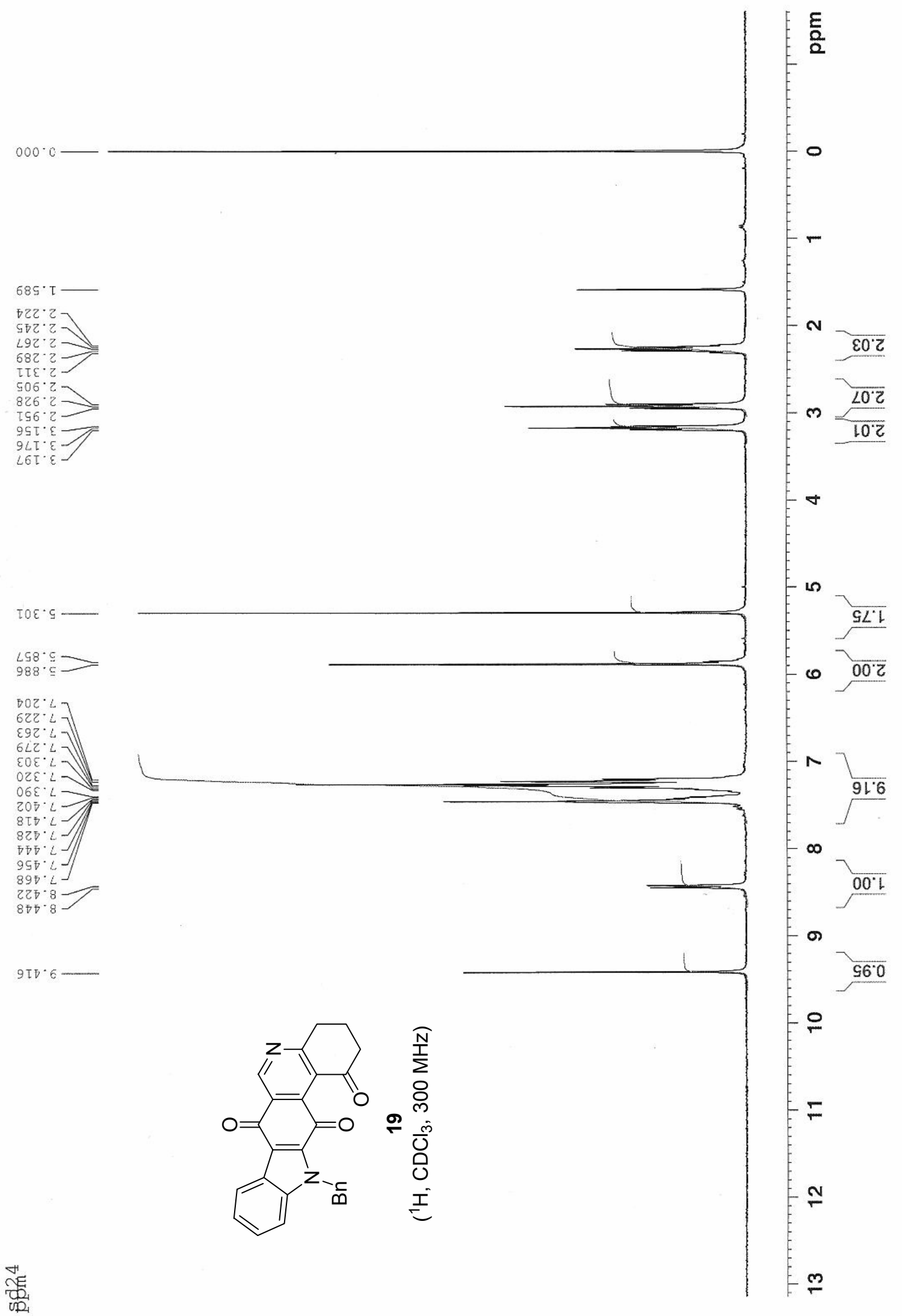


Z๐・ZZ

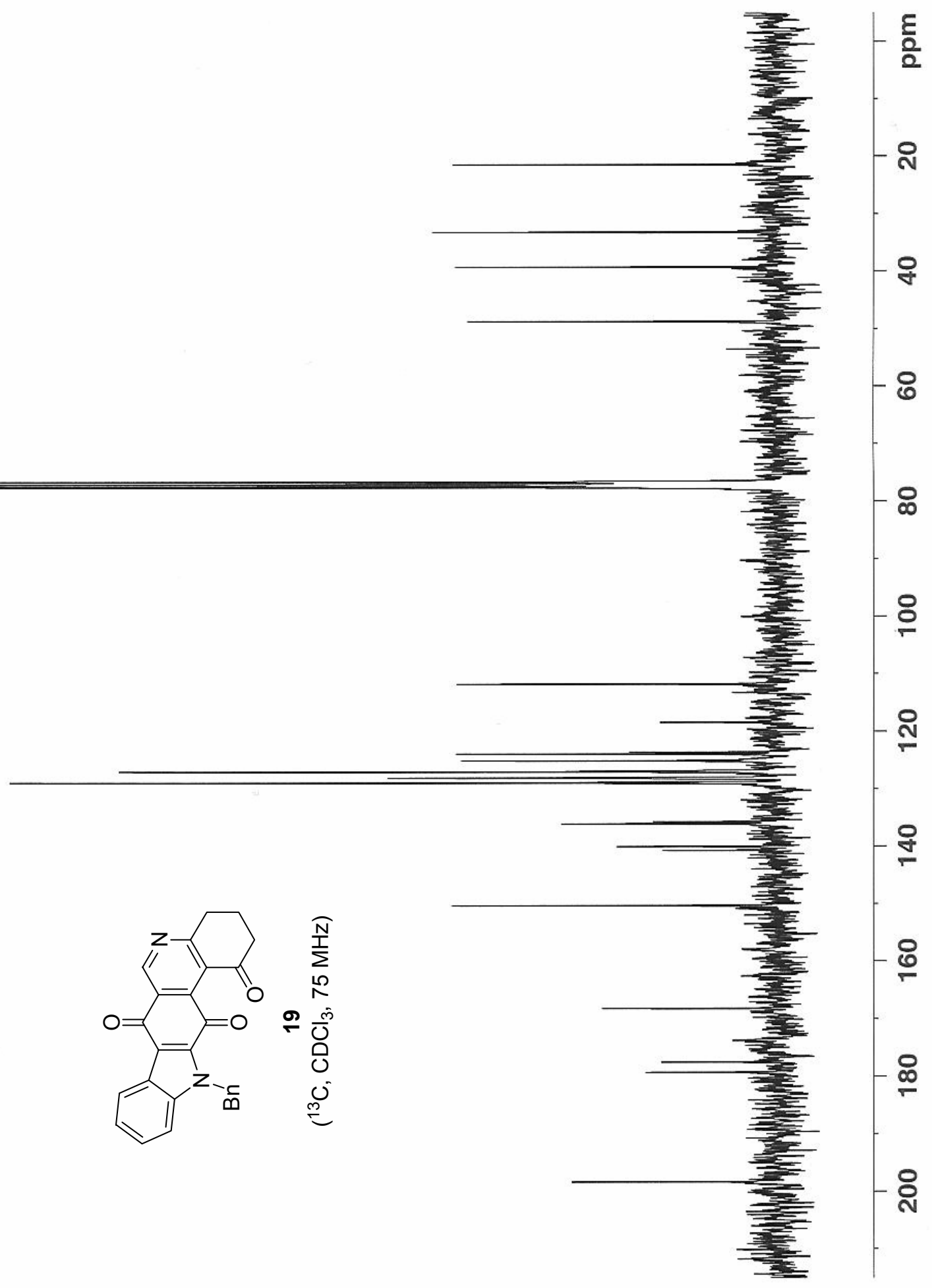

$0 \tau \cdot \varepsilon \varepsilon$

$\angle \tau \cdot 6 \varepsilon$

T9. 87

$\frac{\varepsilon}{2}$

$29 \cdot 9 L$

$70 . \mathrm{LL}$

$97 \cdot L L$ 


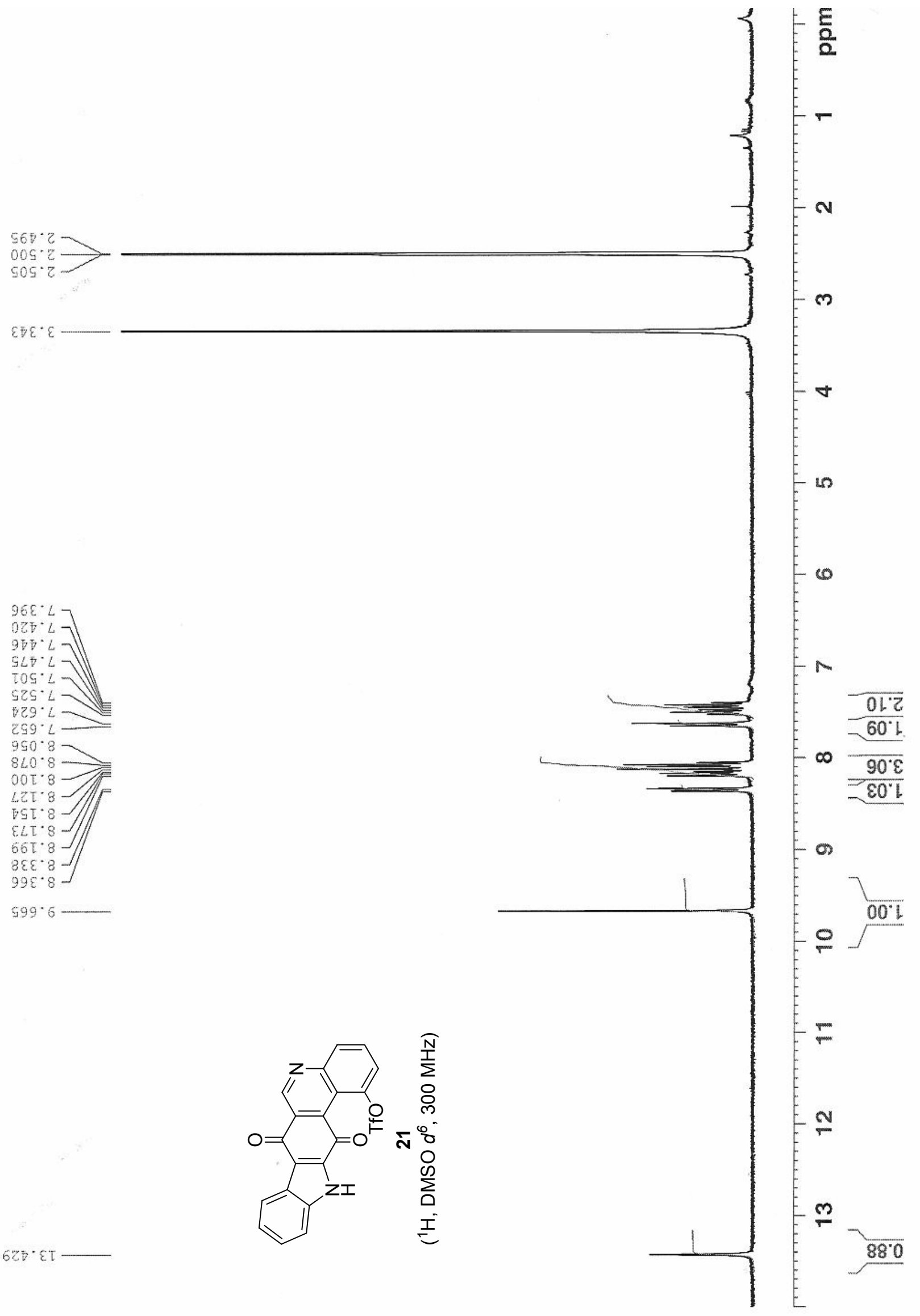




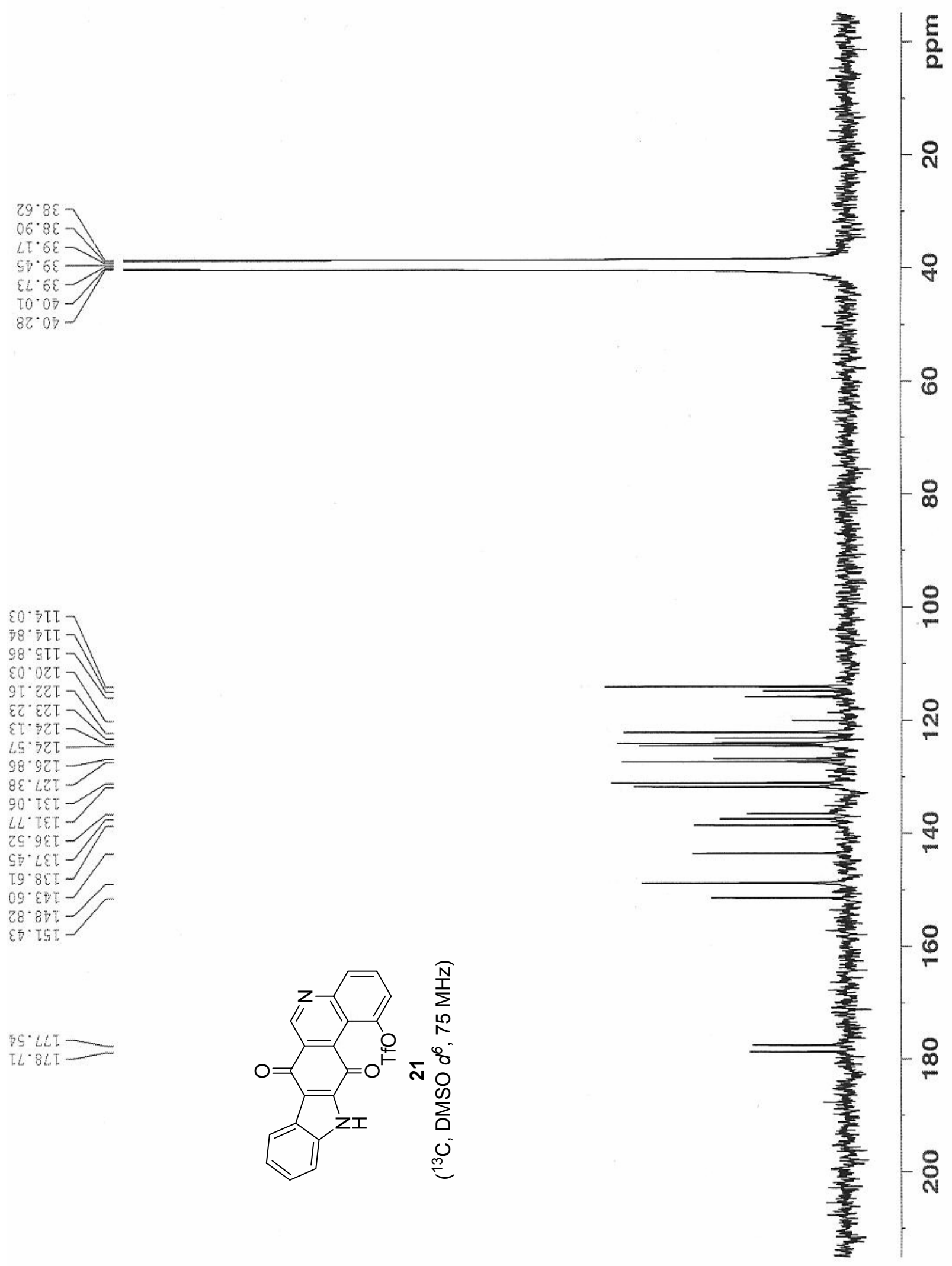



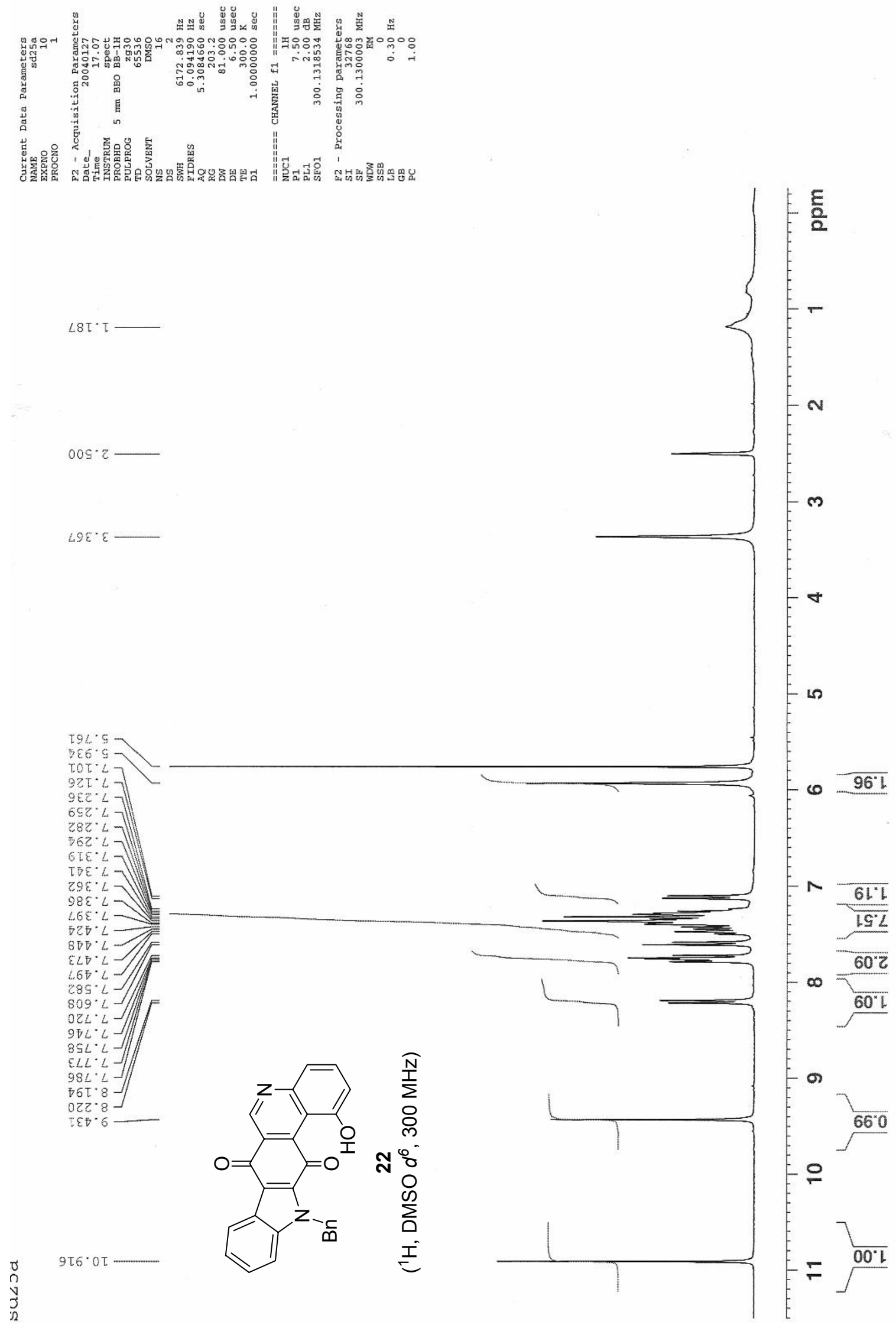

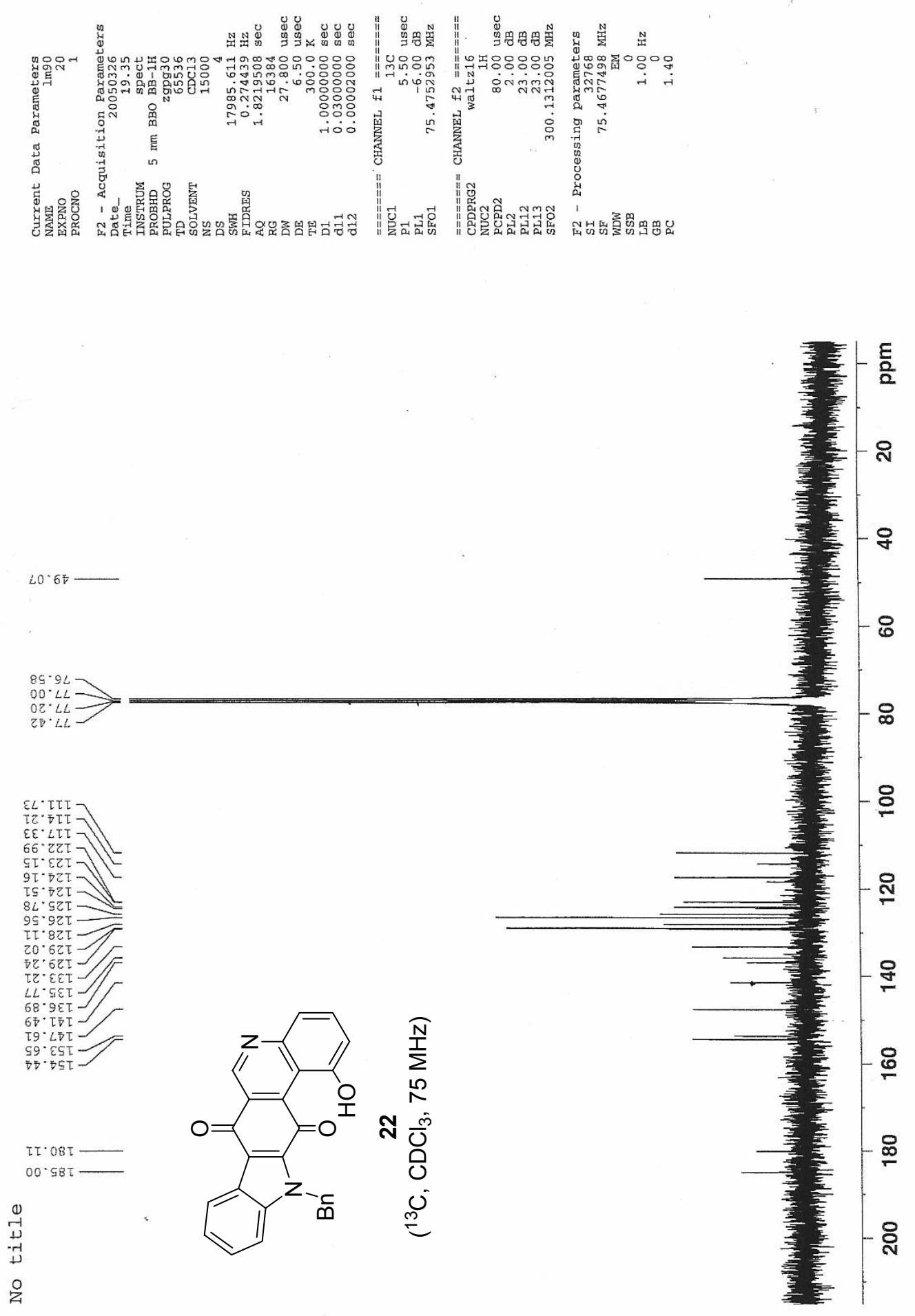


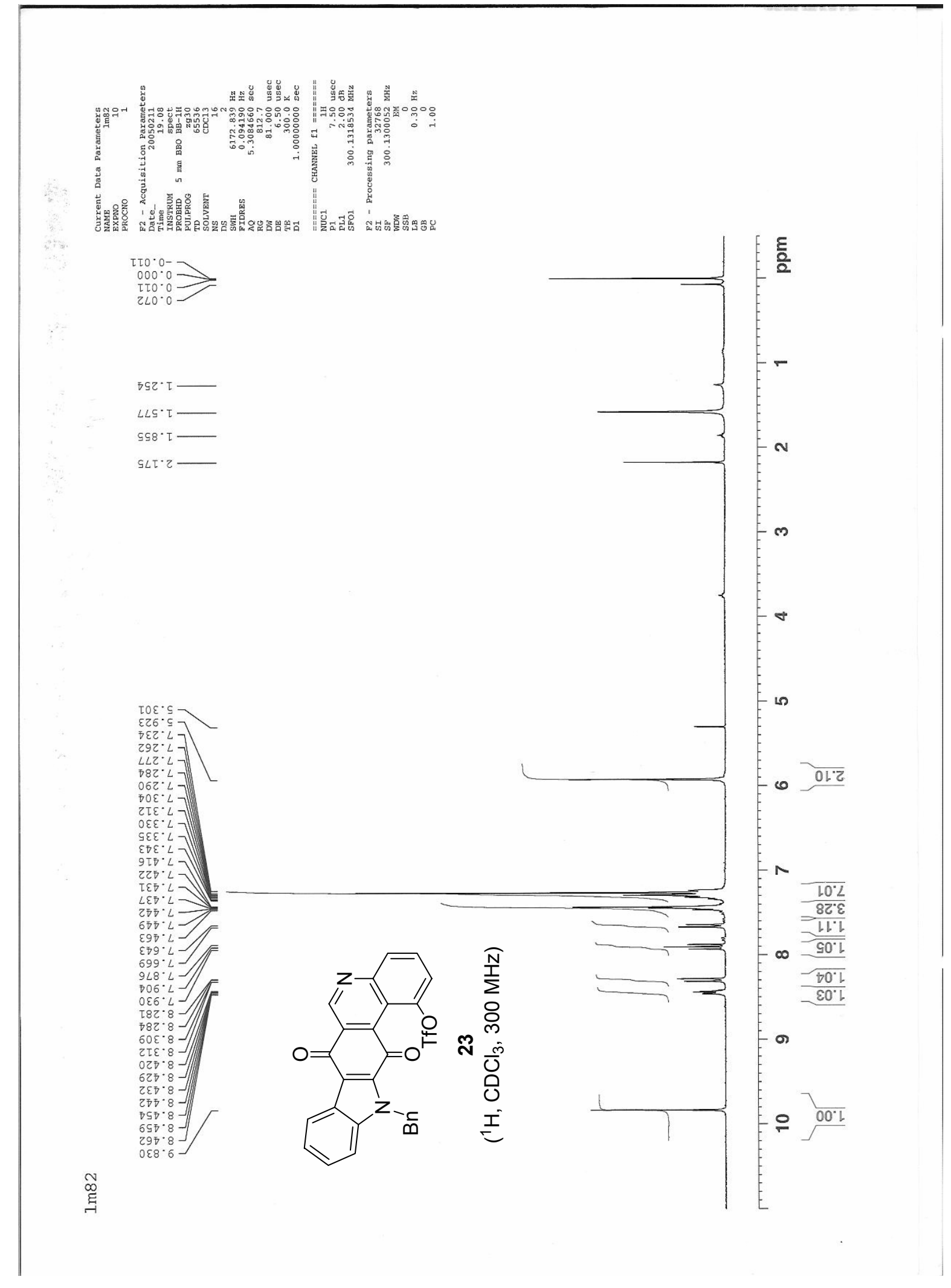



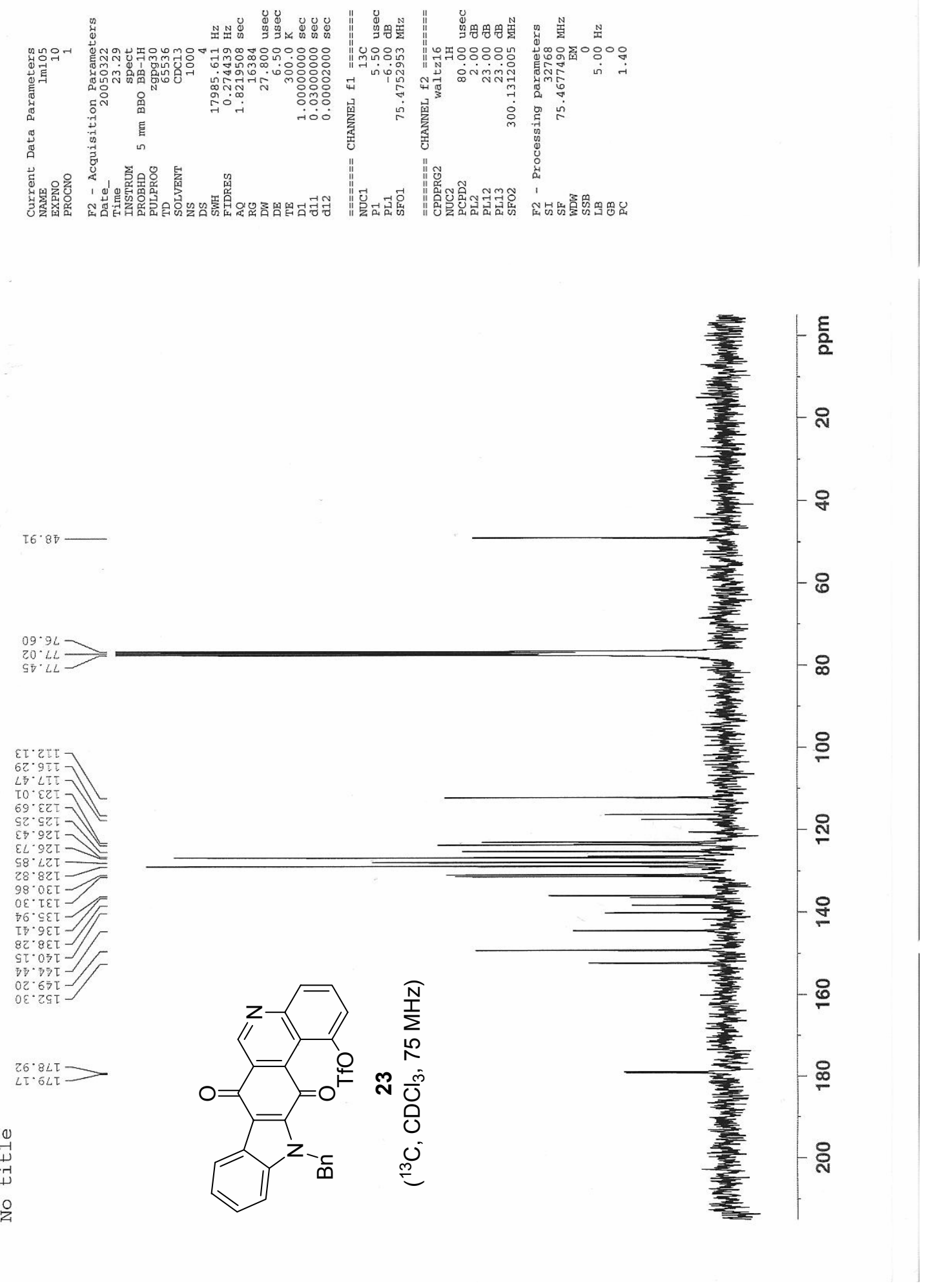


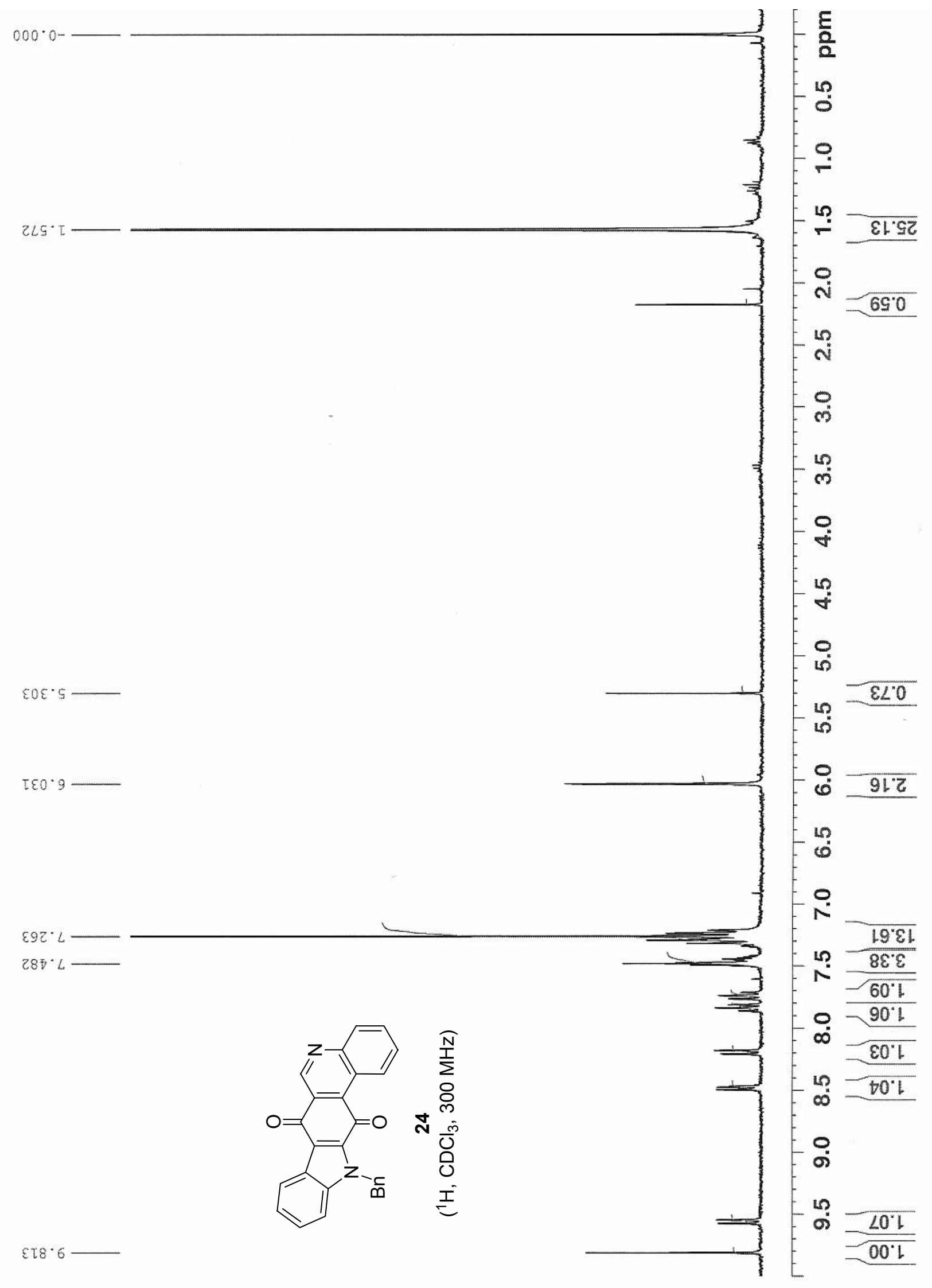



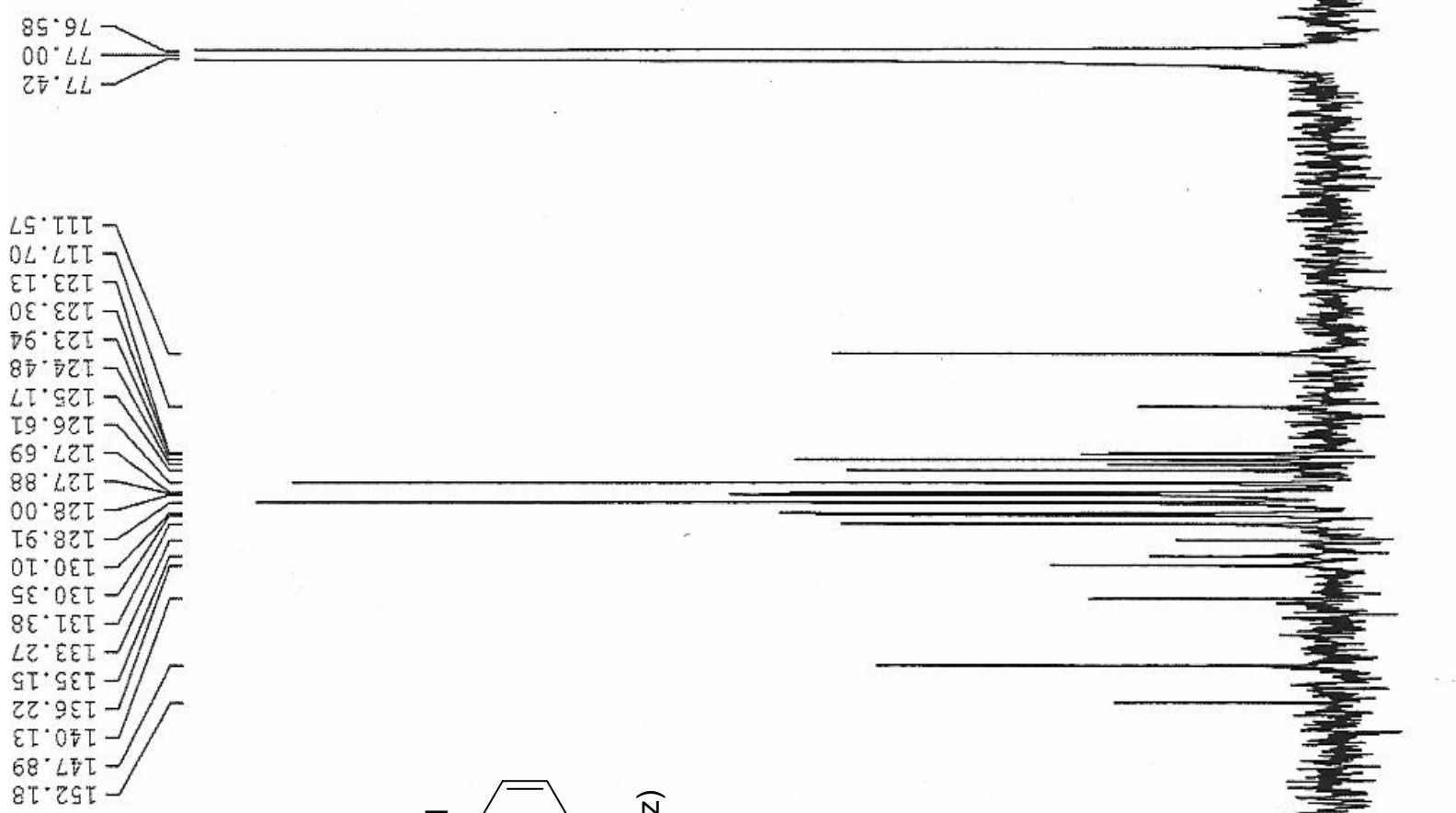

$80^{\circ} \mathrm{T8 \tau}-$
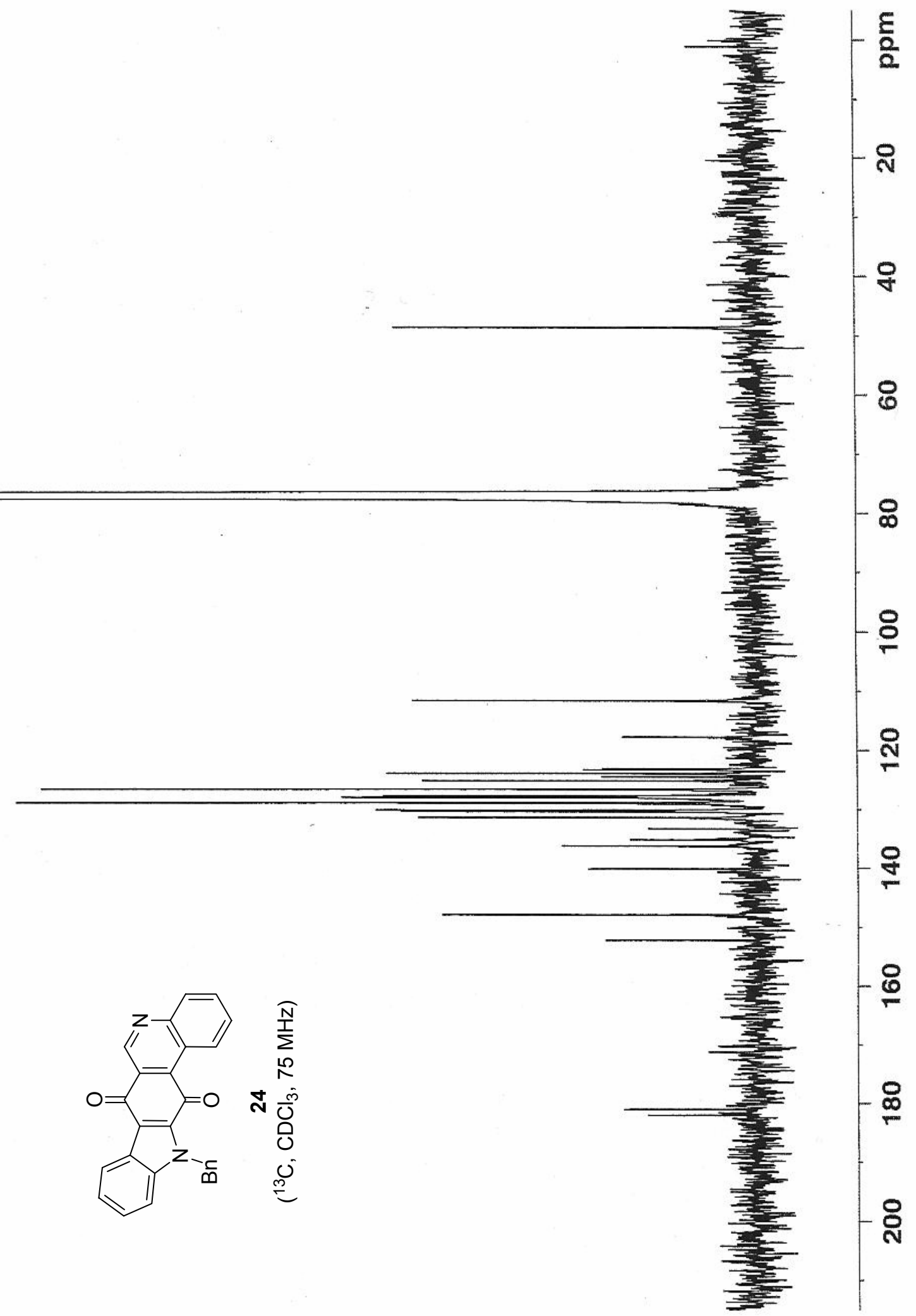